\title{
Brassicaceous roots as an unexpected diversity hot-spot of helotialean endophytes
}

\author{
Jose G. Maciá-Vicente ${ }^{1,2^{*}} \mathbb{D}$, Meike Piepenbring ${ }^{1,2}$ and Ondřej Koukol ${ }^{3}$
}

\begin{abstract}
A high number of fungal strains were isolated from roots of Brassicaceae species collected across western and southern Europe, resulting in an unexpectedly rich collection of Cadophora species. These isolates enable us to present a new and comprehensive view of the ecological, morphological, and phylogenetic traits of root-inhabiting members of this helotialean genus. We provide phylogenetic placement of all of our isolates based on a four-gene dataset, analyze their phenotypic traits in relation to their phylogenetic relationships, and infer the potential distribution ranges of the species by sequence comparisons with available databases. We consider seven well supported phylogenetic lineages as species new to science. Six further lineages probably also represent new species but remain undescribed due to the lack of diagnostic morphological characters. Our results show that Cadophora, as currently circumscribed, is paraphyletic and encompasses a broad spectrum of morphologies and lifestyles. Among the new species, only two (C. ferruginea and C. constrictospora) form phialides and conidia typical of Cadophora, three species (C. echinata, C. gamsii and C. variabilis) produce chains of swollen hyphal segments that may function as holoblastic conidia, and one species (C. fascicularis) produces chains of holoblastic ramoconidia and conidia. Ancestral state reconstruction analysis suggests that phialidic conidiogenesis evolved several times in Cadophora s. lat. from a putatively holoblastic common ancestor. Most Cadophora lineages are rare as estimated from the availability of sequence data, in spite of having relatively wide distribution ranges, whereas five lineages may represent endemic relationships given their restricted distributions. Our dataset, probably the most comprehensive available for Cadophora, nevertheless shows knowledge gaps concerning the phylogenetic relationships within this genus and highlights a need for further investigation.
\end{abstract}

Keywords: 7 new species, Biogeography, Cadophora, Dark septate endophytes, Ploettnerulaceae, Symbiosis

\section{INTRODUCTION}

Species of the genus Cadophora (Ploettnerulaceae, Helotiales, Leotiomycetes) are frequently associated with living plant roots and classified as so-called dark septate endophytes (DSE), a polyphyletic group of non-pathogenic, root-colonizing fungi characterized by melanized hyphae that are conspicuous when observing roots under the microscope (Jumpponen 2001; Sieber 2002; Addy et al.

\footnotetext{
* Correspondence: maciavicente@em.uni-frankfurt.de

${ }^{1}$ Institute of Ecology, Evolution and Diversity, Goethe University Frankfurt, Max-von-Laue-Str. 13, 60438 Frankfurt am Main, Germany

${ }^{2}$ Integrative Fungal Research Cluster (IPF), Frankfurt am Main, Germany Full list of author information is available at the end of the article
}

2005). Cadophora has close phylogenetic affinities with other helotialean lineages with well-known root-associated habit, such as Leptodontidium, Acephala, and Phialocephala (with its teleomorph in Mollisia) - constituting the 'mollisioid clade' sensu Johnston et al. (2019)—and more distantly with mycorrhizal fungi in the Hyaloscyphaceae (Johnston et al. 2019). Whereas Cadophora species are also common as plant pathogens and colonizing substrata other than roots, such as wood or soil (Lagerberg et al. 1927; Bills 2004; Gramaje et al. 2011; Crous et al. 2017), several lines of evidence suggest a degree of adaptation in the genus toward a symbiotic association with plant roots or, leastwise,

(c) The Author(s). 2020 Open Access This article is licensed under a Creative Commons Attribution 4.0 International License, which permits use, sharing, adaptation, distribution and reproduction in any medium or format, as long as you give appropriate credit to the original author(s) and the source, provide a link to the Creative Commons licence, and indicate if changes were made. The images or other third party material in this article are included in the article's Creative Commons licence, unless indicated otherwise in a credit line to the material. If material is not included in the article's Creative Commons licence and your intended use is not permitted by statutory regulation or exceeds the permitted use, you will need to obtain permission directly from the copyright holder. To view a copy of this licence, visit http://creativecommons.org/licenses/by/4.0/. 
the possession of a genomic toolbox favoring the evolution of such a lifestyle. For example, representatives of the genus, or phylogenetically close strains, have been shown to have the ability to promote plant growth (Berthelot et al. 2016), to establish ericoid mycorrhizas (Bizabani and Dames 2015), or to engage in a mycorrhiza-like translocation of phosphorus to the host plant (Almario et al. 2017). Species related to Cadophora also possess genomes enriched in genes encoding carbohydrate active enzymes (CAZymes), proposed as likely markers of a plantassociated lifestyle (Hacquard et al. 2016; Almario et al. 2017; Knapp et al. 2018).

According to the original morphological generic concept, species of Cadophora, with the type species $C$. fastigiata, are characterized by solitary phialides with distinct hyaline, flaring collarettes (Lagerberg et al. 1927). However, deviations from this morphological standard exist, such as $C$. orchidicola with indehiscent conidia produced laterally on undifferentiated hyphae or terminally on unswollen or slightly swollen conidiogenous cells (Currah et al. 1987), or C. antarctica that produces chains of ramoconidia and conidia on holoblastic conidiogenous cells (Crous et al. 2017). The indistinct and largely variable morphological characters have led to significant confusion about the phylogenetic relationships and the boundaries of Cadophora. Soon after its creation, the genus was considered a synonym of Phialophora (Chaetothyriales, Eurotiomycetes) according to similar phialide morphology (Conant 1937), but it was later reinstated as a valid taxon within the Helotiales based on teleomorph links and molecular data (Gams 2000; Harrington and McNew 2003; Day et al. 2012). Other rearrangements of species, into or out of the genus, like those of C. orchidicola (formerly Leptodontidium orchidicola) or Hyaloscypha finlandica (formerly C. finlandica), relied on sequence data and amended morphology-based misplacements (Day et al. 2012; Fehrer et al. 2019). Sequence data have also led to the inclusion of Cadophora in the recently resurrected family Ploettnerulaceae (Jaklitsch et al. 2016; Johnston et al. 2019).

Presently, the genus Cadophora comprises 22 accepted species (www.indexfungorum.org) with diverse ecologies and modes of nutrition. The teleomorph stage is unknown for most Cadophora species, with the exception of several species producing mollisioid apothecia (Harrington and McNew 2003; Day et al. 2012; Pärtel et al. 2017; Ekanayaka et al. 2019). Further recent studies revealed new and hitherto overlooked species colonizing wood necroses (Bien and Damm 2020) and a high phenotypic diversity in the genus mirrored in new discoveries including the psychrotrophic $C$. antarctica isolated from Antarctic soil (Crous et al. 2017); $C$. margaritata, associated with the large poplar longhorn beetle (Linnakoski et al. 2018); and C. helianthi isolated from necrotic tissues in a wilting Helianthus annuus plant (Crous et al. 2019). Due to only subtle differences in morphology from other Phialophora-like fungi, molecular data are essential for species delimitation and identification in the genus.

Plants in the Brassicaceae are primarily nonmycorrhizal (Fitter 2005), but their roots harbor complex communities of endophytic fungi that include a great abundance and diversity of helotialean species, many of which are phylogenetically close to Cadophora (Almario et al. 2017; Glynou et al. 2018a, 2018b; Thiergart et al. 2019; Maciá-Vicente et al. 2020). Often, these Cadophora-like endophytes do not form evident parasitic interactions with the host, as appears to be common in endophytes from other lineages (Durán et al. 2017; Kia et al. 2017, 2018, 2019). Glynou et al. (2016) found a phylotype, identified as Cadophora sp. by ITS rDNA sequence data, to be widespread and abundant within roots of Microthlaspi spp. across western and southern Europe, as well as a number of other related phylotypes with a lower abundance and variable distribution breadths. In spite of their clustering in ITSbased phylotypes based on a $97 \%$ pairwise sequence similarity threshold, isolates in these groups show a high within-phylotype variation in their ITS sequences, their morphology, and their profiles of secondary metabolites production as compared to other lineages of fungal endophytes (Glynou et al. 2017; Maciá-Vicente et al. 2018). Altogether, this variability suggests that these helotialean fungi do not constitute a homogeneous population of widespread and generalist endophytes, but rather comprise a fragmentary array of lineages that have undergone adaptations to local conditions.

Here, we explore the phylogenetic and morphological diversity of 93 Cadophora isolates and related fungi isolated as root endophytes of Microthlaspi spp. (Brassicaceae), and provide descriptions for seven new species. In addition, we test for possible correlations of morphological species traits and ecological characters with phylogenetic relatedness. Lastly, we test the hypothesis that the species represent local (endemic) populations adapted to specific conditions.

\section{METHODS}

\section{Fungal material}

The fungi used in this study were isolated as root endophytes from Microthlaspi spp. plant individuals collected across western and southern Europe (Glynou et al. 2016). The only exception is isolate P6587, which was obtained from roots of Arabidopsis thaliana grown under laboratory conditions on a sterilized substrate containing a $1 \mathrm{~g}$ soil sample from a grassland in The Netherlands (Maciá-Vicente, unpubl.). The isolates were 
selected for their relationship to Cadophora s. lat., based on comparisons of their ITS sequences with the UNITE database of reference fungal sequences (Kõljalg et al. 2005) as described in Glynou et al. (2016), and by BLAST searches against NCBI GenBank. UNITE Species Hypothesis (SH) codes (Kõljalg et al. 2013) were assigned to each isolate using ITS sequence similarities above 99.5\%. Living cultures of all isolates have been deposited in the Integrative Fungal Research (IPF) culture collection hosted at Goethe University (isolate numbers starting with ' $\mathrm{P}$ ' followed by a four-ciphersnumber). A representative subset of the isolates has also been deposited in the CBS culture collection (Westerdijk Fungal Biodiversity Institute, Utrecht, The Netherlands). Dried cultures have been deposited in the Herbarium Senckenbergianum Frankfurt am Main (FR). The details of all isolates are provided in Table S1.

\section{Amplification and sequencing of phylogenetic markers} The ITS sequences for all isolates were available from previous studies (Glynou et al. 2016; Maciá-Vicente, unpublished). In addition, we used aliquots of genomic DNA stored at $-76^{\circ} \mathrm{C}$ from all isolates to obtain partial sequences of the rDNA large (28S) subunit (LSU), the translation elongation factor $1-\alpha($ tef1- $\alpha)$, and the RNA polymerase II second largest subunit (rpb2). The LSU and tef1- $\alpha$ were amplified using primer pairs LROR/LR7 (Hopple Jr and Vilgalys 1994) and EF1-728F/EF1-986R (Carbone and Kohn 1999), respectively, following procedures described by Maciá-Vicente et al. (2016). The $r p b 2$ was amplified using primers RPB2-6F/RPB2-7R (Ashrafi et al. 2018). Purified PCR products were bidirectionally sequenced using the PCR primers at the sequencing laboratory of the Biodiversity and Climate Research Centre (Frankfurt am Main, Germany), or at GATC Biotech (Konstanz, Germany). The assembled sequences have been deposited in GenBank, with accession numbers as listed in Table S1.

\section{Phylogenetic analyses}

Sequences of reference strains from species related to our strains, including species types when available, were retrieved from NCBI GenBank and included in the datasets (Table S2). The sequence datasets for each locus (ITS, LSU, tef1- $\alpha$, and $r p b 2$ ) were first aligned using MAFFT v7.271 (Katoh and Standley 2013) using the GINS-i parameters and manually edited and concatenated in the Geneious software package (Kearse et al. 2012). Genealogical concordance between the ITS, LSU, tef1- $\alpha$, and $r p b 2$ datasets was assessed using $\mathrm{R}$ v3.6.1 ( $\mathrm{R}$ Core Team 2019), by means of the partition homogeneity test (function CADM.global) available in package ape v5.3 (Paradis et al. 2004).
Because no significant support was found for incongruence among the tree topologies resulting from all four loci, a multilocus Maximum Likelihood (ML) phylogeny was obtained with RAxML v8.0 (Stamatakis 2014) using the general time reversible model of nucleotide substitution and the $\Gamma$ model of rate heterogeneity (GTRGAMMA), allowing for different model parameter estimations per partition. The analysis was run on the CIPRES Science Gateway server (Miller et al. 2010) with nonparametric bootstrapping with 1000 replicates used for branch support. A complementary phylogeny based on Bayesian inference (BI) analysis was constructed with MrBayes v3.2.2 (Ronquist et al. 2012). The best-fit substitution model for each of the four loci was determined using the Bayesian information criterion in jModeltest v. 2.1.5 (Darriba et al. 2012). The selected models for the ITS, LSU, tef1- $\alpha$, and $r p b 2$ were TIM $2 \mathrm{ef}+\mathrm{I}+\mathrm{G}, \operatorname{TrN}+$ $\mathrm{I}+\mathrm{G}, \mathrm{HKY}+\mathrm{G}$ and TIM3ef $+\mathrm{G}$, respectively. Two independent MCMC chains were run for $22 \mathrm{M}$ generations with sampling every 100th generation, and a burn-in of $25 \%$ of the sampled trees was used. The average standard deviation of split frequencies estimating convergence reached the level of 0.007 at the end of the analysis. The alignments and trees are available at TreeBASE under accession S25942.

\section{Morphological characterization of isolates}

The selected isolates were first recovered from long-term storage collection tubes containing cultures on corn meal agar (CMA, Sigma-Aldrich, St. Louis, MO, USA) covered with sterilized mineral oil (BDH Chemicals, Ltd., Poole, England), and plated on fresh CMA Petri plates. The isolates were further sub-cultivated on CMA, potato dextrose agar (PDA, AppliChem, Darmstadt, Germany), and 2\% (w/ v) malt extract agar (MEA, Carl Roth, Karlsruhe, Germany), by equidistantly placing on top of each medium three 5mm-diam. plugs taken from two-week-old cultures on CMA. The plates with cultures were incubated upsidedown at $25^{\circ} \mathrm{C}$ in the dark. Three and seven days after inoculation, the diameter of all colonies (three per plate) were measured in two perpendicular directions, and the difference between the measurements at each day were used to calculate the rate of hyphal extension per day. Fifteen days after inoculation, the macro-morphological characteristics of the colonies on different media were recorded, including several measures of the colonies' form, elevation, margin, and color (Table S1).

To estimate the quantity of conidia produced by each isolate, one-month-old cultures on CMA were used, with each Petri plate containing three equidistant colonies as described above. Three six-mm-diam. agar plugs, one per colony, were taken with a cork borer and placed in a $15-\mathrm{ml}$ conical centrifuge tube with $1 \mathrm{ml}$ $0.02 \%(\mathrm{v} / \mathrm{v})$ Tween 20 (BDH Chemicals). The tubes were 
vortexed at maximum speed for $1 \mathrm{~min}$ and then the conidia in suspension were counted in a $10 \mu \mathrm{l}$ aliquot using a haemocytometer. The number of conidia per colony surface area (conidiation) was calculated using the diameter of the agar plugs taken. Calibrated microscopical photographs of the conidia, when present, were captured with a Zeiss Axio Lab.A1 microscope equipped with an Axiocam Erc $5 \mathrm{~s}$ camera, and used to measure the length and width of around 20 conidia per isolate using the software Fiji/ImageJ (Schindelin et al. 2012). The dimensions of conidia were used to calculate conidial volume assuming ellipsoid shapes, according to formula $4 / 3 \pi$ length width ${ }^{2}$.

Isolates representing potentially distinct species based on the phylogenetic analysis were further used for a description of the micro-morphological characters in culture. Sporulating structures on the mycelium were mounted in Melzer's reagent, lactic acid, or lacto-cotton blue and examined using differential interference contrast on an Olympus BX-51 (Olympus, Tokyo, Japan) with a digital camera Olympus DP72 (Olympus). Microscopic measurements are reported as the mean \pm standard deviation of $>30$ measurements.

\section{Ancestral state reconstruction of morphological and ecological characteristics}

An ancestral state reconstruction of the target and reference strains was performed with the $\mathrm{R}$ package phytools v0.5 (Revell 2012), using a ML estimation with an equalrates of transition model. Six discrete micro-morphological and ecological characteristics were considered (Tables S1 and S2): type of conidiogenesis (holoblastic, phialidic, unknown), type of conidia (branched, septate, non-septate, unknown), type of conidial attachment (chains, heads, single, unknown), substrate of origin (leaves, litter, roots, soil, stems, wood, unknown), aquatic lifestyle (yes or no), and life strategy (endophytic, parasitic, saprotrophic, saprotrophic/endophytic, unknown). For each character, the model returns a value of scaled likelihood (SL) per tree node. A subset of the Bayesian consensus tree containing only one specimen per species was used, in which the focal species that did not develop micro-morphological characteristics in culture were excluded. For reference species, data on the morphological and ecological characteristics were compiled from the literature references describing the selected strains (Table S2).

\section{Analysis of isolates' distribution and morphological traits}

Using only the focal isolates of this study, we evaluated whether any of the morphological traits measured were phylogenetically conserved by plotting their values for each lineage in a subset of the phylogenetic tree containing only one set of sequences per species. For quantitative traits (conidiation, conidial length-to-width ratio, growth rate, pigmentation, and conidial volume), the phylogenetic signal for the median values was calculated with the $K$ statistic (Blomberg et al. 2003), using function phylosig of package phytools. The statistic measures conservation of traits among species, with $K=0$ indicating absence of a phylogenetic signal, and $K<1$ or $K>1$ resemblance lower or higher than expected under Brownian motion evolution. Significance of $K$ was assessed by comparing with a random shuffle of values at the tree tips.

We used a variation of principal component analysis (PCA) allowing for a mixture of qualitative and quantitative variables, available in $\mathrm{R}$ package PCAmixdata v3.1 (Chavent et al. 2014), to summarize the isolates' morphological traits and assess their reliability to represent distinct species. Ancestral state reconstruction analysis was used to visualize the distribution of quantitative characters across the isolates' phylogeny, as described above but using a Brownian motion model of character evolution. To investigate the relationship between the isolates' phylogeny, their morphology, and their ecological origins, we first relied on Mantel correlograms (Legendre and Legendre 2012) built with function mantel.correlog of $R$ package vegan v2.5-6 (Oksanen et al. 2015). Cophenetic distances among isolates were extracted from the phylogenetic tree and compared with Euclidean distances calculated from the coordinates of the morphology PCA (see above), or from the geographic coordinates of each location (geographic distance). To assess the relationships with soil physicochemical (soil distance) and climatic (climatic distance) conditions (Table S1), partial Mantel tests were applied to account for covariation with geographic distance.

The data and command line code to reproduce all these analyses is available at https://doi.org/10.6084/m9. figshare.12287816.

\section{Estimation of species' distribution ranges}

The distribution ranges of the species in the isolates' selection were estimated by comparisons against publicly available geographical data on fungal isolations and sequence reads. The ITS sequences of all isolates were first compared using standalone BLAST (blastn version 2.2.31+) searches against a local copy of the NCBI nucleotide (nt) database, containing all fungal ITS sequences in GenBank as of August 2019. A second set of BLAST searches were performed against a selection of NCBI sequence read archive (SRA) objects, representing a selection of high-throughput ITS amplicon sequencing studies of fungi from soils or root samples worldwide, using the function blastn_vdb version $2.6 .1+$ of the NCBI SRA Toolkit (https://trace.ncbi.nlm.nih.gov/ Traces/sra/sra.cgi?view=software). A summary of all 
databases used for these comparisons is provided in Table S3.

In both analyses, records from all BLAST matches with a minimum of $98 \%$ identity, and with a minimum alignment length of $200 \mathrm{nt}$ in the case of SRA objects, were retained. The geographical locations of the records, as latitude/longitude coordinates or countries, were obtained from the sequences' metadata, when available, using custom python scripts. When only data on country but no coordinates were available, the geographical coordinates for the country's centroid were obtained using the $\mathrm{R}$ package maps version 3.3.0 (Becker et al. 2018). The geographical distribution of records was visualized using functions in the R package ggplot2 version 2.3.2.0 (Wickham et al. 2019) and maps of package rnaturalearth version 0.1.0 (South 2017). The command line code for these analyses is available at https://doi.org/10.6 084/m9.figshare.12287816.

\section{RESULTS}

\section{Molecular phylogenetic analyses}

Ninety-three isolates originating as root endophytes from brassicaceous hosts were included in this study. Affinities with members of the helotialean genus Cadophora were recognized based on BLAST comparisons of ITS sequences against the UNITE database (Table S1). Sequences from a selection of 75 strains with reliable species-level identifications were retrieved from NCBI GenBank and used as references for phylogenetic comparison (Table S2). The alignment of partial sequences of ITS, LSU, $r p b 2$, and tef1- $\alpha$ had 2767 characters (638, 885,725 , and 519 , respectively).

Both the ML and BI phylogenetic trees agreed in showing a clear paraphyly in the genus Cadophora, with representative species scattered across four main clades (Fig. 1), e.g. those including C. interclivum, C. meredithiae, and C. gregata; C. luteo-olivacea, C. malorum, and $C$. helianthi; C. orchidicola; and the type species of the genus, C. fastigiata, alongside C. novi-eboraci, $C$. orientoamericana, and C. ramosa; which were interspersed with species of the genera Collembolispora, Mycochaetophora, Oculimacula, Rhexocercosporidium, and Rhynchosporium. Branch support was strong in some of these high-level clades based on both ML bootstrap (BS) and Bayesian posterior probability (PP), although in other cases (e.g., for the cluster containing $C$. interclivum and C. meredithiae) only PP was high (Fig. 1 ). The clade containing the type species $C$. fastigiata appears to be basal to all other clades with Cadophora species, constituting a well-defined clade that henceforth we refer to as Cadophora s. str. Based on the position of the ex-type of Mollisia dextrinospora within Cadophora s. str., we propose its combination into Cadophora.
The focal isolates of this study were grouped into 18 clades with variable branch support (Fig. 1). The multilocus phylogeny showed a better resolution of relationships among isolates than clustering into operational taxonomic units (OTUs) based on 97-98\% ITS sequence similarity, common in diversity barcoding studies, because isolates within each OTU were scattered across distant clades of the tree (Fig. S1). OTUs based on 99\% ITS similarity, however, matched relatively well the clades defined by the multilocus analysis (Fig. S1). Most isolates grouped in two clades, either together with multiple strains of C. interclivum and its sister species $C$. meredithiae (including the ex-type strains) or with two strains (DEPMD S-10 and 1557) tentatively identified as C. orchidicola. The C. interclivum/C. meredithiae clade comprised multiple lineages with medium or no support, including a strongly supported sub-clade formed by isolates P1442 and P2962 that is treated separately in further analyses. In view of phenotypic differences of our isolates with both $C$. interclivum and C. meredithiae, we tentatively named these species $C$. cf. interclivum and $C$. cf. meredithiae. The isolates in the $C$. orchidicola clade, were in turn identified as $C$. cf. orchidicola, given the absence of sequence data for the type specimen of this species. These species-level clades were strongly supported by PP, whereas support was low or only moderately high (89\% in C. cf. orchidicola) based on BS. Other two isolates, P1381 and P2092, were classified as C. luteoolivacea because of their grouping in a lineage including the ex-type sequence of this species (Fig. 1).

The other 13 clades grouped separately from other known species (Fig. 1), and here we propose seven of them as new species (Fig. 1). The remaining six lineages (Cadophora sp. 1, Cadophora sp. 2, Cadophora sp. 3, Cadophora sp. 5, and Mycochaetophora sp.) apparently represent new species but are not described as new here due to the lack of discriminating morphological characters of many cultures which remained sterile even after prolonged cultivation on diverse media.

\section{Ancestral state reconstruction of morphological and ecological characteristics}

The reconstruction of the evolution of three morphological and three ecological characters reflected the paraphyly in Cadophora described above (Fig. 2; Fig. S2). Morphologically, the cluster hosting the genus type $C$. fastigiata (node 5 in Fig. 2) forms a stable group with species that have phialidic conidiogenesis and that produce single non-septate conidia, with the only exception of $C$. bubakii and $C$. margaritata that produce conidia in heads (Fig. 2a-c). Contrarily, the ancestor of all other species (node 2 in Fig. 2) likely had a holoblastic conidiogenesis $(\mathrm{SL}=0.992)$ that was conserved in descendant lineages, with a secondary evolution of phialidic conidiogenesis in the ancestor $(\mathrm{SL}=0.978)$ 


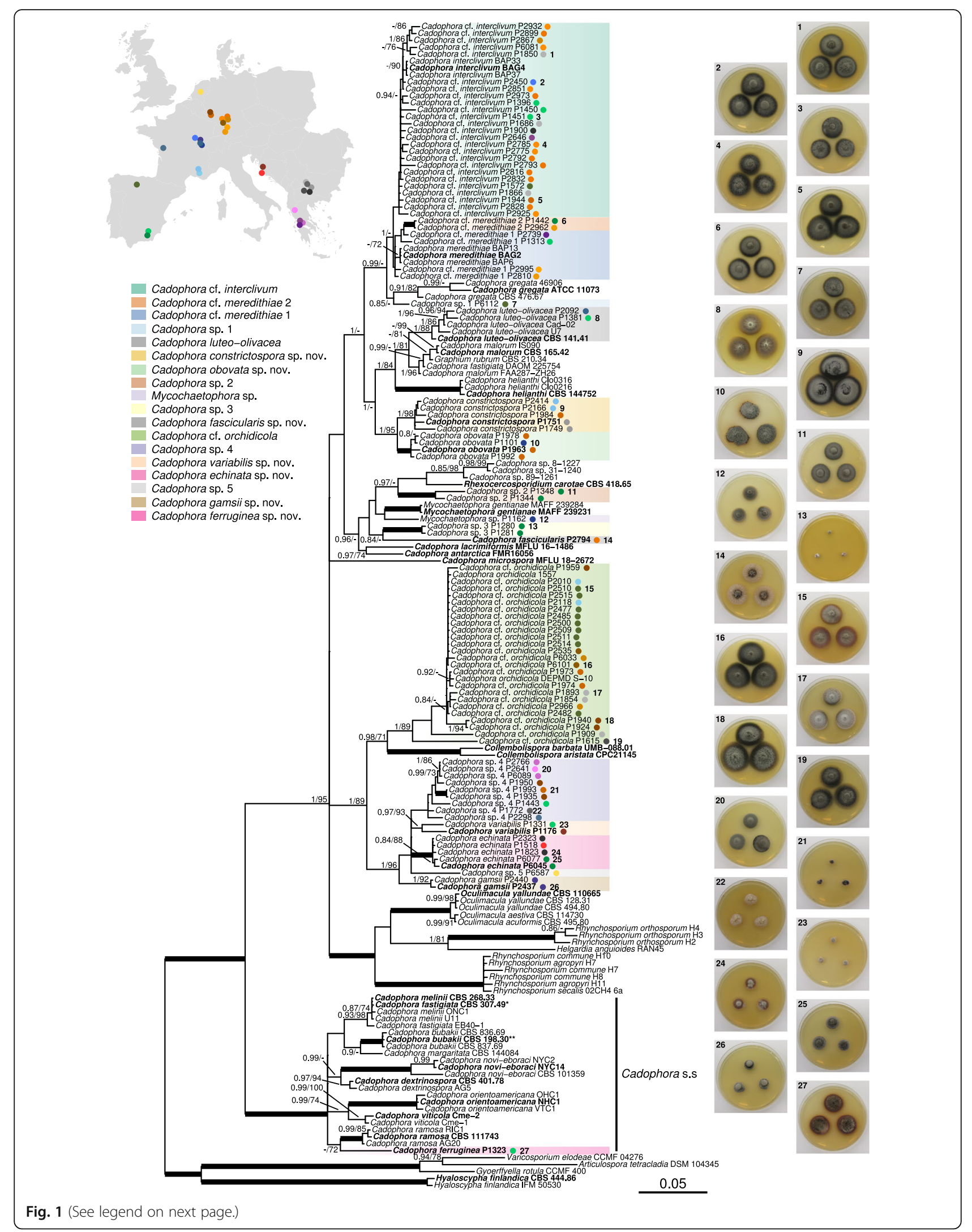


(See figure on previous page.)

Fig. 1 Phylogenetic relationships of the isolates of this study and reference species related to the genus Cadophora. The tree shows a topology based on Bayesian inference analysis of concatenated ITS, LSU, tef1- $a$, and rpb2 partial sequences. Node support values correspond to posterior probabilities (PP) obtained by Bayesian inference, and to bootstrap (BS) analyses based on 1000 replicates of a maximum likelihood phylogeny (PP/BS). Only PP and BS values above 0.8 and 70 are shown, respectively, and thickened branches indicate strong support with PP $=1$ and BS > 99. The isolates of this study are indicated by colored bullets next to the isolates names, with colors representing their geographical origin (see color key in map inset). The species these isolates belong to are highlighted by colored clades (see color key with species names). Strain names highlighted in bold face correspond to species types, or isolates putatively representing them. The Cadophora sensu stricto (s.s.) clade is demarcated with a bracket. Examples of some of the isolates' colony aspect are shown in the pictures rightwards to the tree, depicting 15-dayold pure cultures of individual isolates (see number referring to isolates) on 2\% malt extract agar. *, C. fastigiata CBS 307.49, although not a type specimen, is considered to represent well the genus type by multiple authors (see Discussion). ${ }^{*}$, C. bubakii CBS 198.30 is an isotype

of the clade containing C. malorum, C. luteo-olivacea, $C$. interclivum, and C. gregata (node 6 in Fig. 2a). Whereas the conidial attachment and the type of conidia in this cluster appear to be highly variable across lineages, all species in this cluster may have evolved from an ancestor (node 2 in Fig. 2) with single $(\mathrm{SL}=0.974)$ non-septate conidia $(\mathrm{SL}=$ 0.899).

Ecologically, all Cadophora and allied genera appear to have evolved from a fungus with a terrestrial $(\mathrm{SL}=0.991)$ and most likely parasitic habit $(\mathrm{SL}=0.883$, node 1 in Fig. 2 ), with a subsequent adaptation to aquatic environments only in Collembolispora (also showing branched conidia characteristic of waterborne fungi), and the evolution of saprotrophic and/or endophytic lifestyles in multiple instances (Fig. S2). Whereas it is difficult to establish the substrate preferences of the common ancestor for all Cadophora and allied genera (node 1 in Fig. 2), different lineages apparently radiated to exploit different substrata, mostly plant organs (Fig. 2d), which agrees with the documented plant pathological lifestyle of some Cadophora species. Species of Cadophora s. str. (node 5 in Fig. 2) show a preference towards wood colonization and evolved from a common ancestor that most probably lived on wood ( $\mathrm{SL}=0.999)$; whereas the remaining Cadophora species (node 3 in Fig. 2) may have evolved from a mostly root-colonizing fungus $(\mathrm{SL}=0.883)$, but displaying either adaptations to other substrata, or a flexible lifestyle favoring the colonization of multiple niches (Fig. 2d).

\section{Morphological characters of isolates in culture}

Ordination of isolates according to both quantitative and categorical morphological traits in culture explained a mere $20 \%$ of overall variation, with no clear separation among clades (Fig. S3). This was largely due to the inclusion of categorical variables in the analysis that are not informative for species discrimination. The removal of most categorical characters from the ordination, except for type of conidiogenesis, type of conidia, and type of conidial attachment that appeared to carry phylogenetic signal, increased the proportion of variation explained to $47.7 \%$, discriminating among several of the species (Fig. 3a). Separation among species was strongest based on growth rates in all media and the presence of septa in conidia, and to a lesser extent by the degree of pigmentation on PDA and MEA and conidial volume, explaining differences mainly along the PC 1 axis (Fig. 3b). These drove differences between species like $C$. cf. interclivum and $C$. cf. orchidicola, and slow-growing ones such as Cadophora sp. 3 or C. echinata (Fig. 1a, c; Fig. S4). The type of conidiogenesis, conidial attachment, pigmentation on CMA medium, production of conidia on agar medium, and the conidial length-to-width ratio explained most variation along the PC 2 axis (Fig. 3b), mainly determining differences between the fast-growing species $C$. cf. interclivum and C. cf. orchidicola (Fig. 3a, c; Fig. S4). In spite of the marked differences in quantitative characters between particular species, none of them appeared to be conserved across the phylogeny of the group (Table S4), indicating a lack of strong selective pressure on these traits. However, visualization of the distribution of characters across the isolates' phylogeny showed marked differences between particular clades (Fig. 3c), following the patterns described by the PCA ordination (e.g. fast growth-rates for $C$. cf. interclivum/meredithiae, C. constrictospora, and C. cf. orchidicola, as compared to others; consistently dark pigmentation in C. cf. interclivum; or high conidial length-towidth ratio in $C$. fascicularis). The spores could not be measured for several species that did not produce conidia in culture (see conidiation in Fig. 3c).

\section{Geographic and ecological origins of isolates}

The isolates originated from 30 sampling sites in seven European countries, separated a minimum of $2 \mathrm{~km}$ from one another (Fig. 1). Most clades including the focal isolates comprised isolates originating from geographically distant locations, separated by hundreds to thousands of $\mathrm{km}$, indicating a low level of endemism in the species. Locally restricted distributions were only found in clades represented by individual isolates or by couples, potentially clonal isolates, likely reflecting a low representation in our collection of isolates rather than true geographic endemism. Phylogenetic relatedness between isolates had a low correlation with geographical distance also according to a Mantel test $(r=0.07, P=0.01)$, indicating a lack of spatial structure in these fungal populations. Likewise, partial Mantel tests accounting for spatial distance showed 


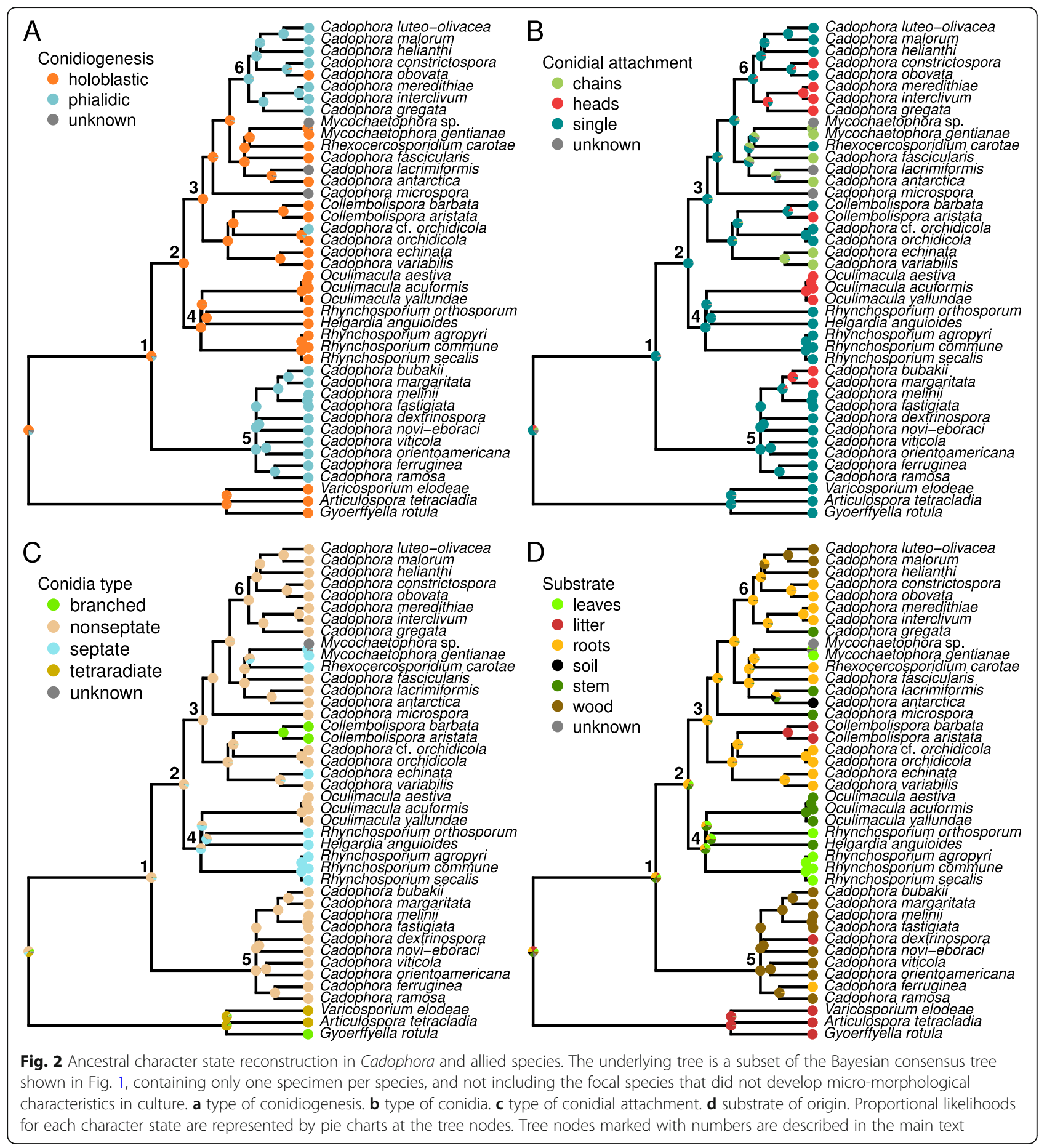

that similarity in climatic factors or in physico-chemical characteristics of soils of isolation sites were poorly associated with phylogenetic similarity among isolates $(r=0.08$, $P=0.047$ and $r=0.05, P=0.089$, respectively).

\section{Species distribution ranges}

Comparison of ITS sequences from all isolates with GenBank records and sequences from high-throughput amplicon sequencing studies of fungal diversity worldwide (Table S3) showed potential distribution ranges largely restricted to the northern hemisphere (Fig. 4, Fig. S5). We evaluated BLAST results with a minimum of $97 \%$ sequence similarity to account for undefined ITS-based species delimitations, but only values above $99 \%$ are likely to approximate actual species boundaries (Fig. S1), with $100 \%$ values providing conservative assignments. At $100 \%$ 


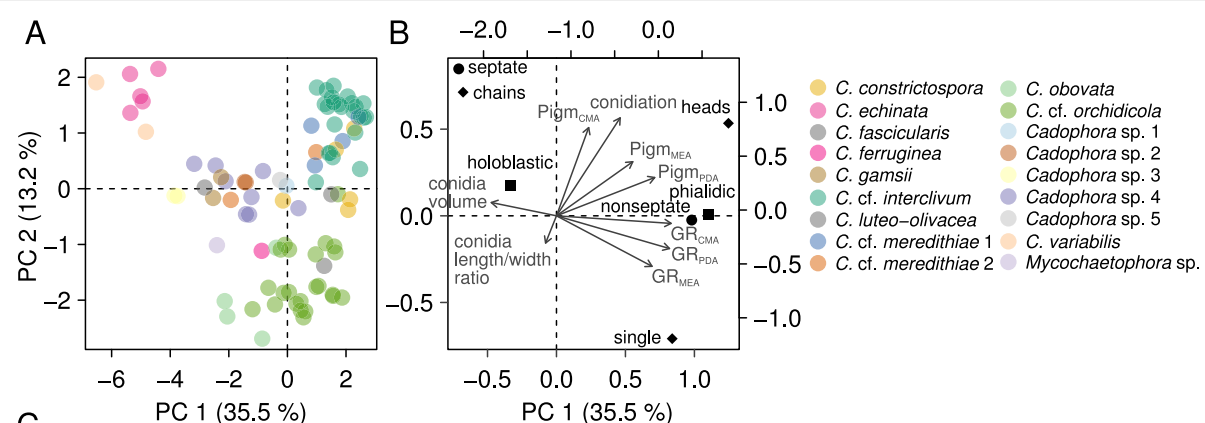

C

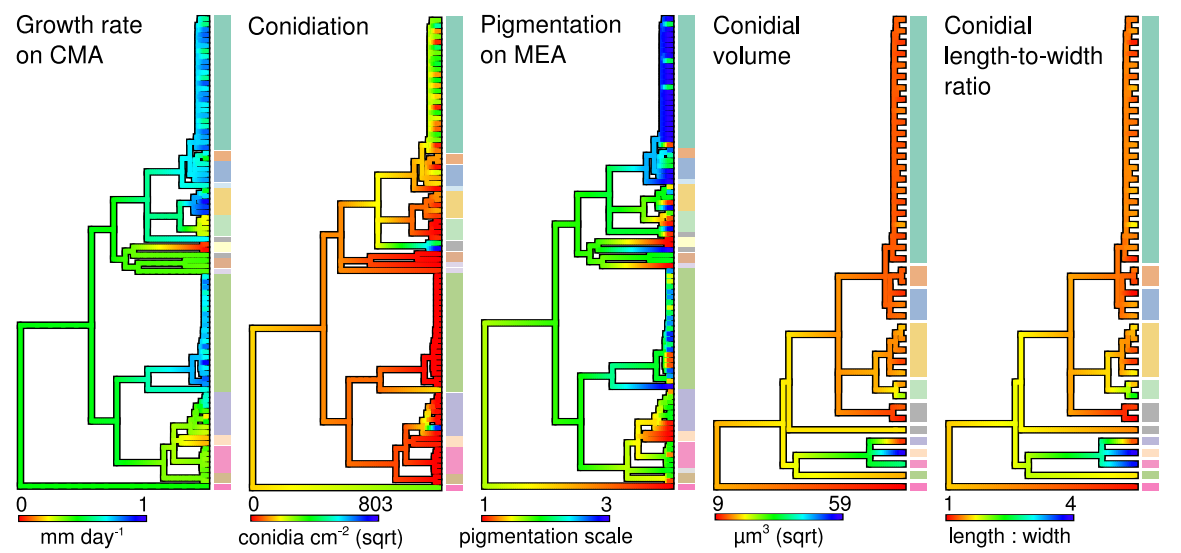

Fig. 3 Morphological characterization of the isolates of this study. a Principal component analysis (PCA) ordination of isolates according to their quantitative morphological characters. Each point represents one isolate, with color indicating the species to which it belongs to. $\mathbf{b}$ PCA scores showing the contribution of each morphological character to the separation of the isolates, as indicated by the direction and magnitude of the arrows. c Distribution of selected quantitative characters across the isolates' phylogeny. Colors next to tree tips indicate the isolates' species (see color key). Note that trees for conidial volume and conidial length-to-width ratio contain only a subset of the species that produced conidia in culture. For trees showing all the characters included in b, see Fig. S3. Abbreviations: GR, growth rate on each agar medium (indicated by subscript); Pigm, degree of dematiaceous pigmentation on each agar medium (indicated by subscript)

similarity, most species showed a restricted European distribution, with matches both in the nt and SRA databases spanning a maximum of $5000 \mathrm{~km}$ (Fig. 4b, Fig. S5). Only C. obovata, C. cf. orchidicola, Cadophora sp. 1, Cadophora sp. 4, and C. variabilis surpassed that threshold, with reports in either North America and/or Asia, but never south from the equator (Fig. 4b, Fig. S5). In contrast to these species, C. fascicularis, C. ferruginea, Cadophora sp. 3, Cadophora sp. 5, and Mycochaetophora sp. showed very restricted ranges with respect to GenBank records, often even at low percent identities (Fig. 3b, Fig. S4).

\section{TAXONOMY}

Cadophora dextrinospora (Korf) Koukol \& MaciáVicente, comb. nov.

MycoBank: MB 834822.

Basionym: Mollisia dextrinospora Korf, Mycotaxon 10: 462 (1980).
Note: The affinity of $M$. dextrinospora, distinct by dextrinoid reaction of its ascospores, to the genus Cadophora, and notably to C. fastigiata, was recognized already by Greenleaf and Korf (1980) and later confirmed by Gams (2000). The combination into Cadophora is further supported by the current placement of Mollisia in a lineage distinct from Ploettnerulaceae (Jaklitsch et al. 2016), and its connection with Phialocephala (Tanney et al. 2016).

Cadophora constrictospora Koukol \& Maciá-Vicente, sp. nov. - Figs. 1, 5 .

MycoBank: MB 834823.

Etymology: After the production of secondary conidia that are distinctly constricted.

Diagnosis: Cadophora constrictospora differs from C. fastigiata in having distinctly constricted secondary conidia. 

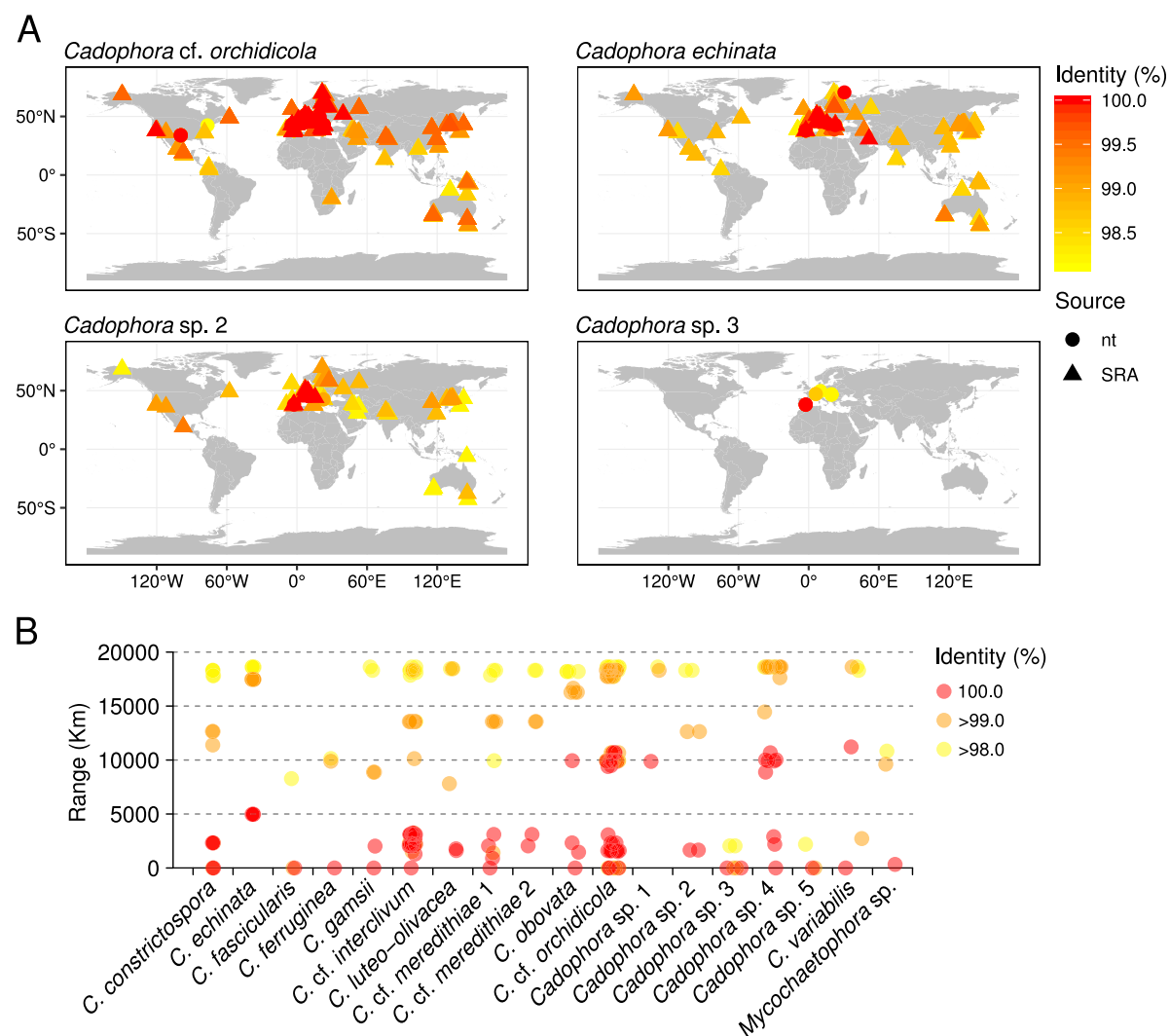

Fig. 4 Potential worldwide distribution of the Cadophora species target of this study as inferred by BLAST comparisons against the NCBI GenBank's nucleotide database (nt), and selected high-throughput ITS amplicon sequencing datasets available at the sequence reads archive (SRA; Table S3). a Maps showing the worldwide distribution of BLAST matches for selected Cadophora species (see Fig. S4 for maps with all the species in this study). Each map shows the BLAST search results for all the isolates within each species. Points represent the geographic locations of BLAST matches, either from the nt or the SRA database (indicated by point shape). Only BLAST matches with percent identity above $97 \%$ are shown, with points color indicating percent identity value. b Species distribution ranges based on BLAST results at 98, 99, or 100\% percent identity. Ranges correspond to the farthest distance between two BLAST matches in the map, at each percent identity. For each species, each point corresponds to the range calculated for each isolate within the species

Type: Bulgaria: Nepraznentsi, 42.67 N 022.84 E, 740 m a.s.l., endophytic in roots of Microthlaspi sp., 14 May 2013, T. Ali \& S. Ploch [isol. K. Glynou] (FR 0255157 holotype; P1751 = CBS 146371 - ex-type cultures: GenBank accessions: ITS $=$ KT269023, LSU $=$ MN339369, tef1 $-\alpha=$ MN325874, $r p b 2=$ MN367280).

Description: In culture - Colonies on MEA reaching 28-30 mm diam after $10 \mathrm{~d}$, on PDA and CMA reaching $27-28 \mathrm{~mm}$ and $17-18 \mathrm{~mm}$ diam, respectively. $M y$ celium septate, hyphae subhyaline to pale brown, forming fascicles up to $18 \mu \mathrm{m}$ diam, hyphae $2-4 \mu \mathrm{m}$ thick. Conidiophores pale brown, straight, unbranched, producing terminal or intercalary fascicles of phialides, rarely absent. Conidiogenous cells phialidic, pale brown, smooth, lanceolate, with distinct collarette up to $3.5 \mu \mathrm{m}$ long, $(8.5-) 10-14.5(-17.5) \times 2-4 \mu \mathrm{m}$ wide. Conidia of two types: primary conidia hyaline, ellipsoid to narrowly ellipsoid, smooth, nonseptate, with distinct projection on the basis, forming compact heads on the top of phialides, $4-6.5 \mu \mathrm{m}$ long and 2$2.5 \mu \mathrm{m}$ wide, the mean conidium length/width ratio 2.3:1; secondary conidia pyriform, spathulate to ossiform secondary conidia with distinct constrictions in the middle are formed in older cultures, 6.5-10(12.5) $\times 3-5 \mu \mathrm{m}$ wide, the mean conidium length/width ratio $2: 1$.

Note: The newly described species is morphologically most similar to $C$. fastigiata that has phialides and conidia of similar shape and size, however C. constrictospora produces also large secondary conidia with distinct constriction in cultures more than $30 \mathrm{~d}$ old. These secondary conidia do not seem to have distinct origin, so that we assume that they are produced by ageing phialides. The morphological similarity is however result of convergence, since the two species are largely unrelated (Fig. 1). 


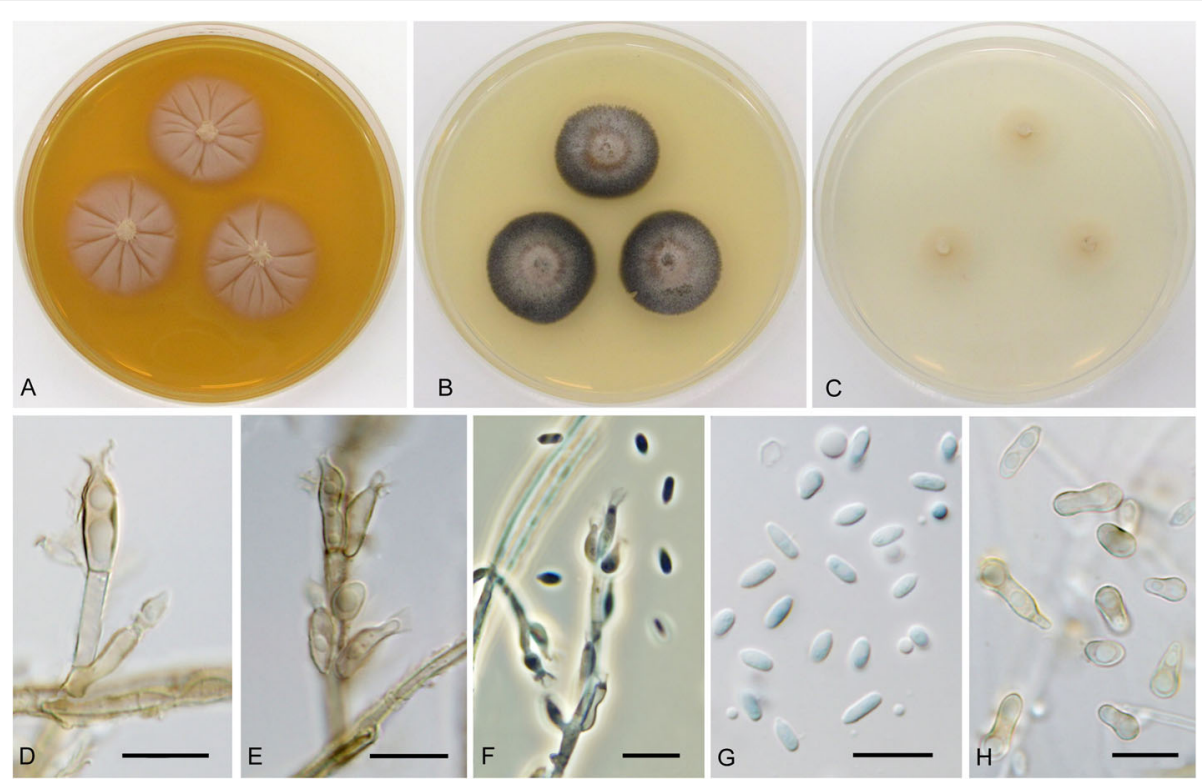

Fig. 5 Cadophora constrictospora (CBS 146371 - ex-type culture). a-c 15-day-old colonies on MEA, PDA, and CMA, respectively. d-e conidiophore with terminal and intercalary phialides. $\mathbf{f}$ conidiophore with phialides and conidia in phase contrast. $\mathbf{g}$ primary conidia. $\mathbf{h}$ secondary conidia. Bars: $10 \mu \mathrm{m}$

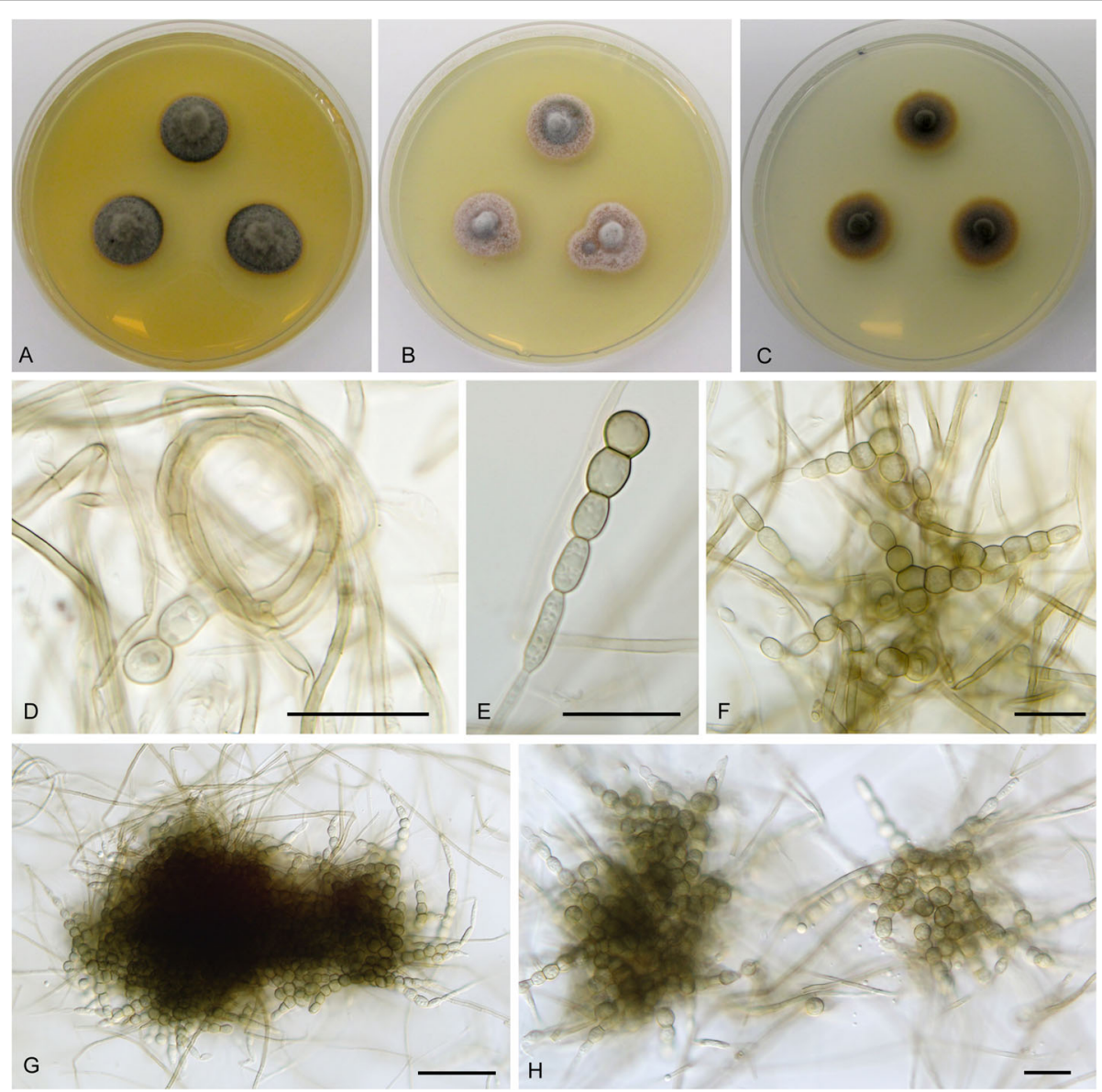

Fig. 6 Cadophora echinata (CBS 146383 - ex-type culture). a-c, 15-day-old colonies on MEA, PDA, and CMA, respectively. d young conidium formed on hyphal coil. e terminally formed conidium. $\mathbf{f}-\mathbf{h}$ complex conidia at various stages of maturity. Bars: $\mathrm{D}-\mathrm{E}=10 \mu \mathrm{m}, \mathrm{F}-\mathrm{H}=20 \mu \mathrm{m}$ 
Cadophora echinata Koukol \& Maciá-Vicente, sp. nov. - Figs. 1, 6.

MycoBank: MB 834828.

Etymology: After the frequent production of conidia having echinate appearance.

Diagnosis: Cadophora echinata is distinct in producing complex holoblastic conidia that branch into compact echinate bodies up to $150 \mu \mathrm{m}$ large.

Type: Spain: Puebla de Don Fadrique, 38.05 N 002.54 W, 1612 m a.s.l., endophytic in roots of M. perfoliatum, 2 May 2013, J.G. Maciá-Vicente (FR 0255199 - holotype; P6045 = CBS 146383 - ex-type cultures; GenBank accessions: ITS = KT270239, LSU $=$ MN339428, tef1 $\alpha=\mathrm{MN} 325932, r p b 2=$ MN367267).

Description: In culture - Colonies on MEA reaching 15 $\mathrm{mm}$ diam after $10 \mathrm{~d}$, on PDA and CMA reaching 14$16 \mathrm{~mm}$ and $15-16 \mathrm{~mm}$ diam, respectively. Mycelium septate, hyphae hyaline to dark brown, rarely forming coils up to $38 \mu \mathrm{m}$ diam, hyphae $2-3 \mu \mathrm{m}$ wide. Conidiophores not developed. Conidiogenous cells integrated, terminal, holoblastic, monoblastic, smooth, cylindrical, 3-5 $\mu \mathrm{m}$ wide. Conidia holoblastic complex, originating as chains of fusiform, oval to almost globose cells strongly constricted at the septa, pale brown and becoming darker towards the centre, later branching repeatedly and forming compact tuft-like bodies up to $150 \mu \mathrm{m}$ diam, individual cells $5.5-9 \times 5-9 \mu \mathrm{m}$ wide.
Additional material studied: Croatia: Gospić, $44.46 \mathrm{~N}$ 015.40 E, $755 \mathrm{~m}$ a.s.l., endophytic in roots of M. erraticum, 4 May 2013, T. Ali [isol. K. Glynou] (FR 0255154; P1518 - culture). - Bulgaria: Divlya, 42.57 N 022.69 E, $685 \mathrm{~m}$ a.s.l., endophytic in roots of $M$. erraticum, 14 May 2013, T. Ali \& S. Ploch [isol. K. Glynou] (FR 0255159; P1823 - culture); ibidem (FR 0255182; P2323 - culture). - Spain: Puebla de Don Fadrique, 38.05 N 002.54 W, $1612 \mathrm{~m}$ a.s.l., endophytic in roots of $M$. perfoliatum, 2 May 2013, J.G. Maciá-Vicente (FR 0255200; P6077 culture).

Note: For comments on C. echinata see the note under C. gamsii.

Cadophora fascicularis Koukol \& Maciá-Vicente, sp. nov. - Figs. 1, 7.

MycoBank: MB 834824.

Etymology: After the production of ramoconidia in apical fascicles.

Diagnosis: Morphologically similar to $C$. antarctica, but with conidiophores with holoblastic cylindrical conidiogenous cell producing chains of ramoconidia and conidia.

Type. Germany: Darstadt, 49.68 N, 010.00 E, 278 m a.s.l., endophytic in roots of M. erraticum, 6 June 2013, K. Glynou \& J.G. Maciá-Vicente [isol. K. Glynou] (FR 0255219 - holotype; P2794=CBS 146382 - ex-type

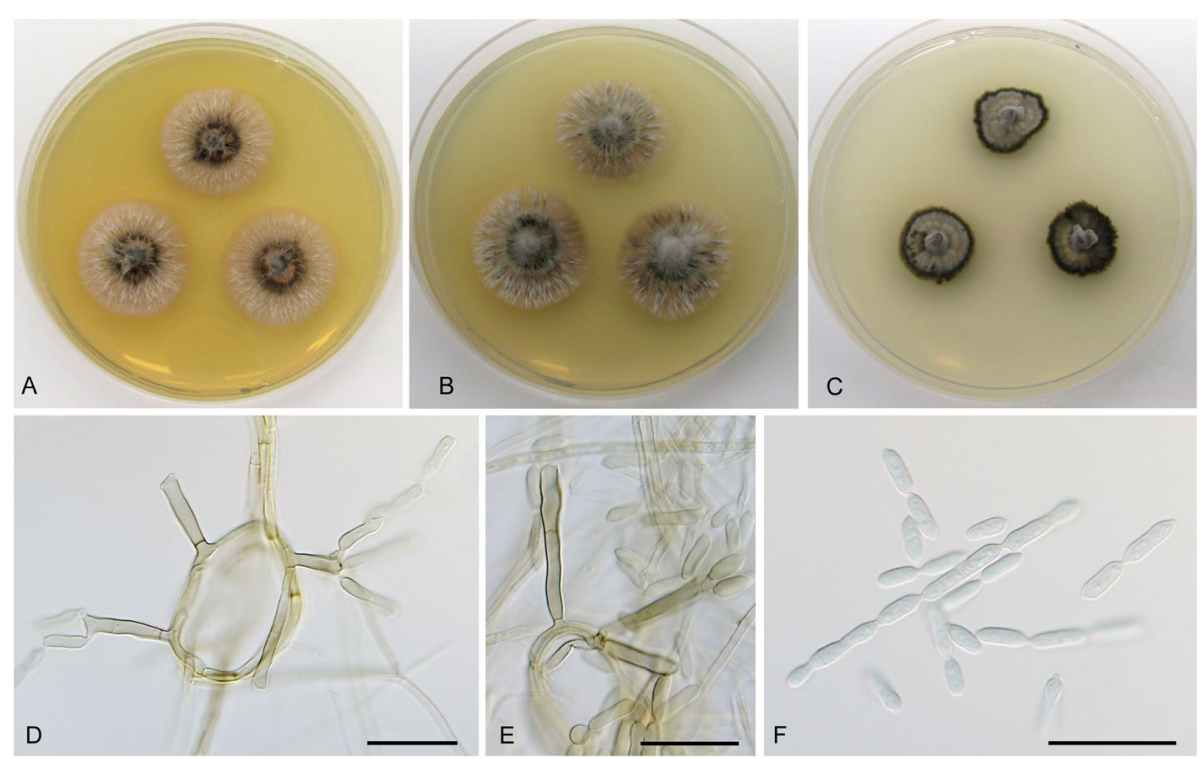

Fig. 7 Cadophora fascicularis (CBS 146382 - ex-type culture). a - c 15-day-old colonies on MEA, PDA, and CMA, respectively. d - e conidiophores formed on hyphal coils. $\mathbf{f}$ chains of conidia and ramoconidia. Bars: $20 \mu \mathrm{m}$ 
cultures; GenBank accessions: ITS $=$ KT269992, LSU = MN339414, tef1- $\alpha=$ MN325918).

Description: In culture - Colonies on MEA reaching 17$18 \mathrm{~mm}$ after $10 \mathrm{~d}$, on PDA and CMA reaching 16-17 $\mathrm{mm}$ and $14-15 \mathrm{~mm}$, respectively. Mycelium septate, hyphae hyaline to dark brown, frequently forming coils up to $50 \mu \mathrm{m}$ in diam, hyphae $2-2.5 \mu \mathrm{m}$ in diam. Conidiophores growing from vegetative hyphae or hyphal coils, straight, smooth, consisting of 1-2 cells terminated by a conidiogenous cell, up to $50 \mu \mathrm{m}$ long and $3-4 \mu \mathrm{m}$ wide, frequently reduced to the conidiogenous cell. Conidiogenous cells holoblastic, polyblastic, smooth, cylindrical, cuneiform to almost club shaped with several loci at the apex, (11)12.5-18(20.5) $\mu \mathrm{m}$ long and up to $3-5 \mu \mathrm{m}$ wide in the upper part. Ramoconidia holoblastic $0(-1)$ septate, pale brown to hyaline, sometimes paler towards the apex, usually forming fascicles on the top of the conidiogenous cell, smooth, straight to slightly curved, subcylindrical to fusiform, not constricted at the septum, (6.5)10.5-16(20) $\mu \mathrm{m}$ long and 2.5-4 $\mu \mathrm{m}$ wide. Conidia hyaline, smooth, elongated lemon shaped to fusiform with slight constriction in the middle, forming short chains, (6.5) $8-11(15) \mu \mathrm{m}$ long and $2-3 \mu \mathrm{m}$ wide, the mean conidium length/width ratio 3.5:1.

Note: Cadophora fascicularis differs from most Cadophora species by a lack of phialidic conidiogenous cells. It is morphologically somewhat comparable to C. antarctica that also produces chains of ramoconidia and conidia on holoblastic conidiogenous cells. However, C. fascicularis has well developed conidiophores, ramoconidia, and conidia are more elongated than in C. antarctica.

Cadophora ferruginea Koukol \& Maciá-Vicente, sp. nov. - Figs. 1, 8.

MycoBank: MB 834826.

Etymology: After the production of reddish globules and mycelium.

Diagnosis: Morphologically distinct from C. fastigiata in having smaller phialides with indistinct collarette and reddish mycelium in culture.

Type: Spain: Puebla de Don Fadrique, 38.04 N 002.48 W, $1630 \mathrm{~m}$ a.s.l., endophytic in roots of $M$. perfoliatum, 2 May 2013, J.G. Maciá-Vicente (FR 0255144 - holotype; P1323 = CBS 146363 - ex-type cultures; GenBank accessions: $\quad$ ITS $=$ KT268618,$\quad$ LSU $=$ MN339356,$\quad$ tef1 $-\alpha=$ MN325861).

Description: In culture - Colonies on MEA reaching 18$20 \mathrm{~mm}$ diam after $10 \mathrm{~d}$, on PDA and CMA reaching 18$19 \mathrm{~mm}$ and $16-22 \mathrm{~mm}$ diam, respectively. Mycelium septate, hyphae subhyaline to reddish, forming fascicles up to $15 \mu \mathrm{m}$ diam, hyphae $2-4 \mu \mathrm{m}$ wide. Conidiophores hyaline, straight, branched and producing terminal fascicle of phialides, rarely absent. Conidiogenous cells phialidic, hyaline, smooth, with indistinct collarette (that may disappear at older phialides), $6-10 \times 2-3 \mu \mathrm{m}$ wide.

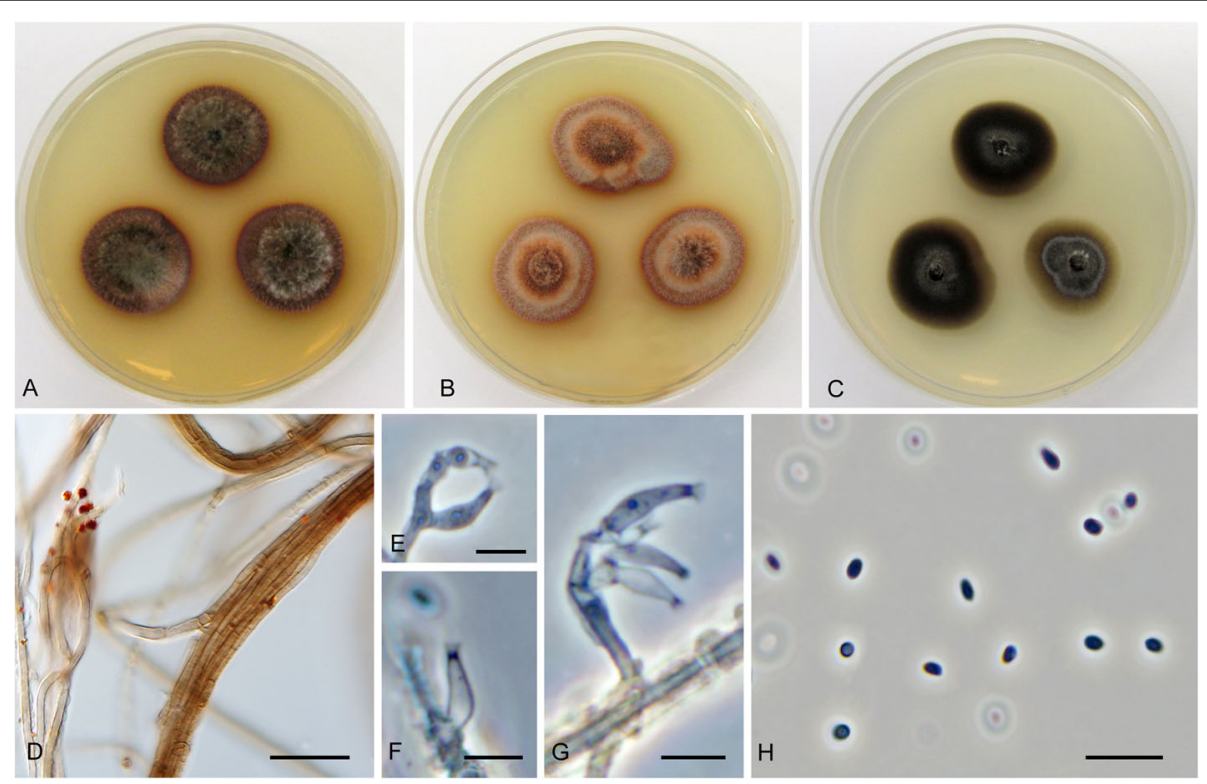

Fig. 8 Cadophora ferruginea (CBS 146363 - ex-type culture). a - c 15-day-old colonies on MEA, PDA, and CMA, respectively. d hyphal fascicles. e g, phialides in phase contrast. $\mathbf{h}$, conidia in phase contrast. Bars: $D=20 \mu \mathrm{m}, \mathrm{E}-\mathrm{G}=5 \mu \mathrm{m}, \mathrm{H}=10 \mu \mathrm{m}$ 


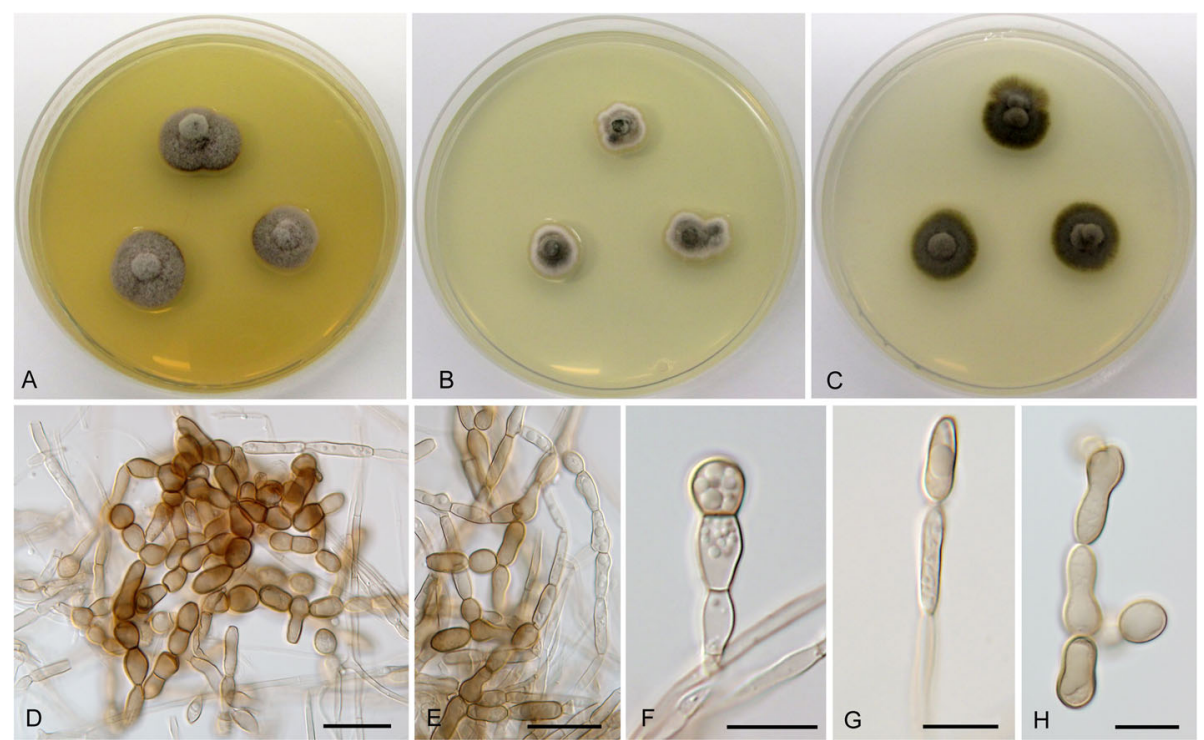

Fig. 9 Cadophora gamsii (CBS 146379 - ex-type culture). a - c, 15-day-old colonies on MEA, PDA, and CMA, respectively. d, chains of predominantly thick walled conidia. e, chains of both thick and thin walled conidia. $\mathbf{f}-\mathbf{g}$, terminally produced conidia. $\mathbf{h}$, detached conidia. Bars: $D-E=20 \mu m, F-H=10 \mu m$

Conidia hyaline, ellipsoid, smooth, nonseptate, forming compact heads on the top of phialides $3-5 \times 1.5-2.5 \mu \mathrm{m}$ wide, the mean conidium length/width ratio 1.8:1.

Note: Cadophora ferruginea belongs among other phialidic members of this genus, but produces phialides that are generally smaller and may be distinguished by reddish mycelium.

Cadophora gamsii Koukol \& Maciá-Vicente, sp. nov. Figs. 1, 9.

MycoBank: MB 834825.

Etymology: Named in honour of Walter Gams (19342017) who contributed significantly to the study of the genera Cadophora and Phialophora.

Diagnosis: Morphologically distinct from C. echinata by long and rarely branched chains of variably shaped conidia.

Type: France: Malbrans, 47.11 N 006.07 E, 543 m a.s.l., endophytic in roots of $M$. erraticum, 4 May 2013, A.K. Buch E X. Xia [isol. K. Glynou] (FR 0255184 - holotype; P2437 = CBS 146379 - ex-type cultures; GenBank accessions: $\quad$ ITS $=\mathrm{KT} 269668, \quad$ tef1 $-\alpha=\mathrm{MN} 325899, \quad r p b 2=$ MN367272).

Description: In culture - Colonies on MEA reaching 12$16 \mathrm{~mm}$ diam after $10 \mathrm{~d}$, on PDA and CMA reaching 11 $\mathrm{mm}$ and $12-13 \mathrm{~mm}$ diam, respectively. Mycelium septate, hyphae hyaline to pale brown, hyphae $1.5-2.5 \mu \mathrm{m}$ wide. Conidiophores not developed. Conidiogenous cells integrated, terminal, holoblastic, monoblastic, smooth, cylindrical, 2.5-5 $\mu \mathrm{m}$ wide. Conidia pale brown rather thin walled to dark brown, thick walled, smooth, mostly non-septate (rarely 1-septate), rather variable in shape, from globose to pyriform, spathulate, fusiform, with distinct constriction in the middle when elongated, forming long chains that are rarely branched; thin walled elongated conidia (10-)14-20($22.5) \times 3.5-5.5 \mu \mathrm{m}$ wide (when 1-septate $20 \mu \mathrm{m}$ long), mean conidium length/width ratio $3.9: 1$; thick walled conidia 7-14.5(-18.5) $\times 5-9 \mu \mathrm{m}$ wide, mean conidium length/width ratio 1.7:1.

Additional material studied: France: Malbrans, $47.11 \mathrm{~N}$ 006.07 E, $543 \mathrm{~m}$ a.s.l., endophytic in roots of $M$. erraticum, 4 May 2013, A.K. Buch \& X. Xia [isol. K. Glynou] $(\mathrm{P} 2440=$ CBS $146379-$ cultures $)$.

Note: Cadophora gamsii produces long chains of conidia of variable shapes similarly to $C$. echinata, where these chains branch and may form large sclerotia-like bodies. However, these structures may be also interpreted as inflated hyphal segments (chlamydospores). In C. orchidicola they are formed together with regular conidia. These structures presumably do not disperse very far, but rather survive close by and so may have a dormancy function similar to the microsclerotia of dothidealean fungi (Tsuneda et al. 2001). 


\section{Cadophora cf. interclivum - Figs. 1, 10.}

Description: In culture - Colonies on MEA reaching 16$17 \mathrm{~mm}$ diam after $10 \mathrm{~d}$, on PDA and CMA reaching 17$18 \mathrm{~mm}$ and $17-18 \mathrm{~mm}$ diam, respectively. Mycelium septate, hyphae hyaline to dark brown, frequently forming coils, hyphae $2-2.5 \mu \mathrm{m}$ wide. Conidiophores straight, bearing single or multiple phialides arranged in intercalary or terminal fascicles. Conidiogenous cells phialidic, slightly darker, $6-10 \mu \mathrm{m}$ long and $2-3 \mu \mathrm{m}$ wide in the upper part terminated by a collarette $2-3.5 \mu \mathrm{m}$ long. $\mathrm{Co}-$ nidia ellipsoid to ovate with slightly prominent basis, hyaline, with smooth wall, $3-4.5 \times 2-3 \mu \mathrm{m}$ wide, forming clusters but not slimy heads, mean conidium length/ width ratio 1.4:1.

Specimens examined: Germany: Darstadt, 49.68 N 010.00 E, 278 m a.s.l., endophytic in roots of M. erraticum, 6 June 2013, K. Glynou \& J.G. Maciá-Vicente [isol. K. Glynou] (FR 0255220; P2973 - culture). - Spain: Puebla de Don Fadrique, $38.04 \mathrm{~N} 002.48 \mathrm{~W}, 1630 \mathrm{~m}$ a.s.l., endophytic in roots of M. perfoliatum, 2 May 2013, J.G. Maciá-Vicente [isol. K. Glynou] (FR 0255149; P1396 - culture). Germany: Darstadt, 49.68 N 010.00 E, 278 m a.s.l., endophytic in roots of M. erraticum, 6 June 2013, K. Glynou \& J.G. Maciá-Vicente [isol. K. Glynou] (P2816 - culture).

Note: In total, 25 strains clustered with three sequences of C. interclivum (including the ex-type strain). Both phenotypic and molecular characteristics were difficult to interpret in concordance with the description of $C$. interclivum by Walsh et al. (2018). Our strains produced slightly darker Phialocephala-like phialides with distinct wide open collarettes at the tip of straight conidiophores (Fig. 9), i.e. different from cylindrical conidiogenous cells with cylindrical collarettes and conidia aggregated in slimy heads of C. interclivum (Walsh et al. 2018). In analyses of individual genetic loci, only sequence data of the intron in the tefl- $\alpha$ gene enabled to distinguish two clades referring to $C$. interclivum and C. meredithiae with medium support ( $\mathrm{PP}=0.87$, data not shown). In another analysis, the strains were mixed and no clades were clearly delimited. A similar phenomenon was recorded by Walsh et al. (2018), who found two well supported lineages only when analyzing sequences of genes coding the RNA polymerase II largest subunit (rpb1) and $\beta$-tubulin. Unfortunately, we did not amplify these markers. Obviously, the lineage C. interclivum/C. meredithiae contains multiple, hitherto unrecognized root colonizing species, and commonly used markers, such as ITS or $r p b 2$, have limited ability to distinguish them.

Cadophora obovata Koukol \& Maciá-Vicente, sp. nov. - Figs. 1, 11.

\section{MycoBank: MB 834827.}

Etymology: After the obovate shape of conidia.

Diagnosis: Morphologically distinct in having obovate conidia produced from monoblastic phialide-like conidiogenous cells.

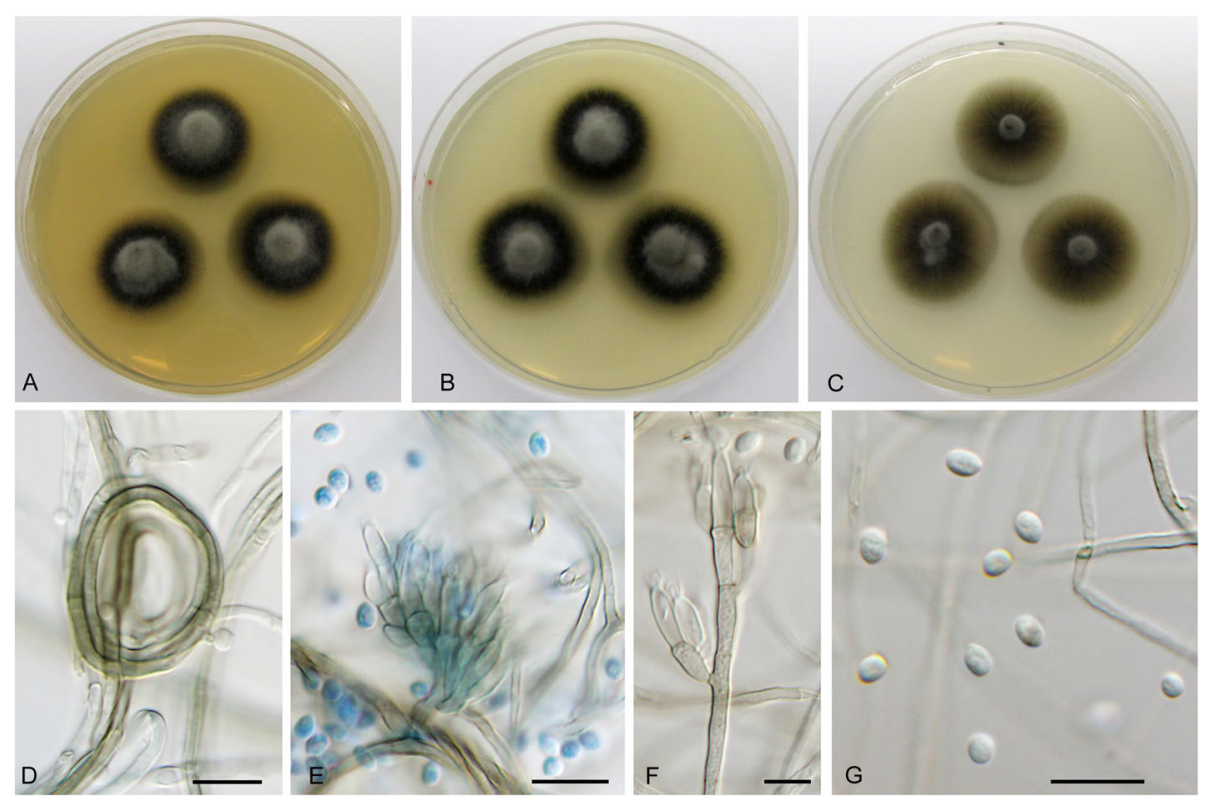

Fig. 10 Cadophora cf. interclivum (isolate P2973). a - c 15-day-old colonies on MEA, PDA, and CMA, respectively. d hyphal coil. e fascicle of phialides with conidia (mounted in lactic acid with cotton blue). f, terminal and lateral fascicles of phialides. g, conidia. Bars: $10 \mu \mathrm{m}$ 


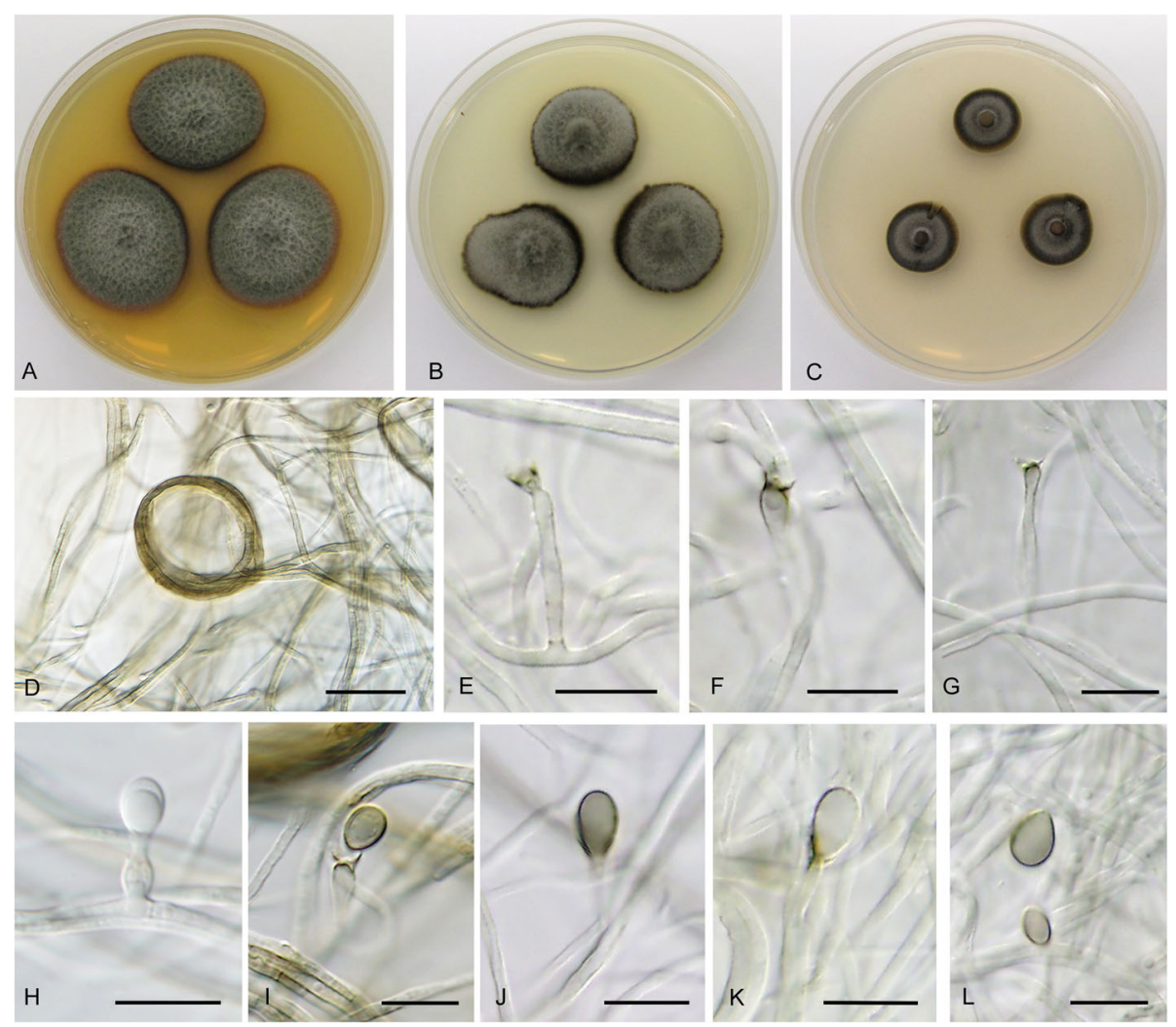

Fig. 11 Cadophora obovata (CBS 146374 - ex-type culture). a - c 15-day-old colonies on MEA, PDA, and CMA, respectively. $\mathbf{d}$ hyphal coil. e - $\mathbf{g}$ conidiogenous cells. $\mathbf{h}$ young conidiogenous cell producing conidium. $\mathbf{i}$ conidiogenous cell releasing conidium. $\mathbf{j}-\mathbf{I}$ conidia still attached to conidiogenous cell or free. Bars: $D=20 \mu m, E-L=10 \mu m$

Type: Germany: Ettringen, 50.37 N 007.22 E, 504 m a.s.l., endophytic in roots of $M$. erraticum, 9 May 2013, $M$. Thines [isol. K. Glynou] (FR 0255171 - holotype; P1963 = CBS 146374 - ex-type cultures; GenBank accessions: $\quad$ ITS $=$ KT269230, $\quad$ LSU $=$ MN339384, tef1 $-\alpha=$ MN325888, $r p b 2=$ MN367298).

Description: In culture - Colonies on MEA reaching 27$30 \mathrm{~mm}$ diam after $10 \mathrm{~d}$, on PDA and CMA reaching 22$25 \mathrm{~mm}$ and $15 \mathrm{~mm}$ diam, respectively. Mycelium septate, hyphae hyaline to dark brown, frequently forming coils or hyphal fascicles $15 \mu \mathrm{m}$ thick, hyphae $1.5-2.5 \mu \mathrm{m}$ wide. Conidiophores absent. Conidiogenous cells holoblastic, monoblastic, obconical, $17-22 \mu \mathrm{m}$ long and $2-3 \mu \mathrm{m}$ wide in the upper part terminated by a collarette 2$3.5 \mu \mathrm{m}$ long. Conidia obovate, pale grey-brown, with smooth wall, $6-8(-9.5) \times 3.5-6 \mu \mathrm{m}$, mean conidium length/width ratio $1.5: 1$.

Additional material examined: France: Saint-GeorgesArmont, 47.41 N 006.56 E, 285 m a.s.l., endophytic in roots of M. erraticum, 19 April 2013, J.G. Maciá-Vicente (FR 0255142; P1101 = CBS 146359 - cultures) Germany: Ettringen, $50.37 \mathrm{~N} 007.22$ E, $504 \mathrm{~m}$ a.s.l., endophytic in roots of $M$. erraticum, 9 May 2013, M. Thines [isol. K. Glynou] (FR 0255174; P1978 - culture); ibidem (FR 0255176; P1992 - culture).

Note: This newly described species is distinct from other Cadophora species in having a putatively monoblastic conidiogenous cell. Although the cell resembles a phialide in having a basal swelling of the conidiogenous cell and an irregular collarette-like structure, no subsequent enteroblastic conidiogenesis typical for phialides was observed. Giving its position in the phylogeny, this type of conidiogenous cell seems to represent a retrogression of enteroblastic phialidic conidiogenesis.

\section{Cadophora cf. orchidicola - Figs. 1, 12.}

Description: In culture - Colonies on MEA reaching 30 mm diam after $10 \mathrm{~d}$, on PDA and CMA reaching 32-33 $\mathrm{mm}$ and $29-30 \mathrm{~mm}$ diam, respectively. Mycelium septate, hyphae subhyaline to dark brown, smooth to verrucose, occasionally forming coils and chains of inflated hyphal segments, rarely branched, $6 \mu \mathrm{m}$ thick, hyphae $1.5-2.5 \mu \mathrm{m}$ wide. Conidiophores straight or flexuous, hyaline, branched or unbranched, bearing single conidiogenous cells intercalary or multiple arranged in terminal 


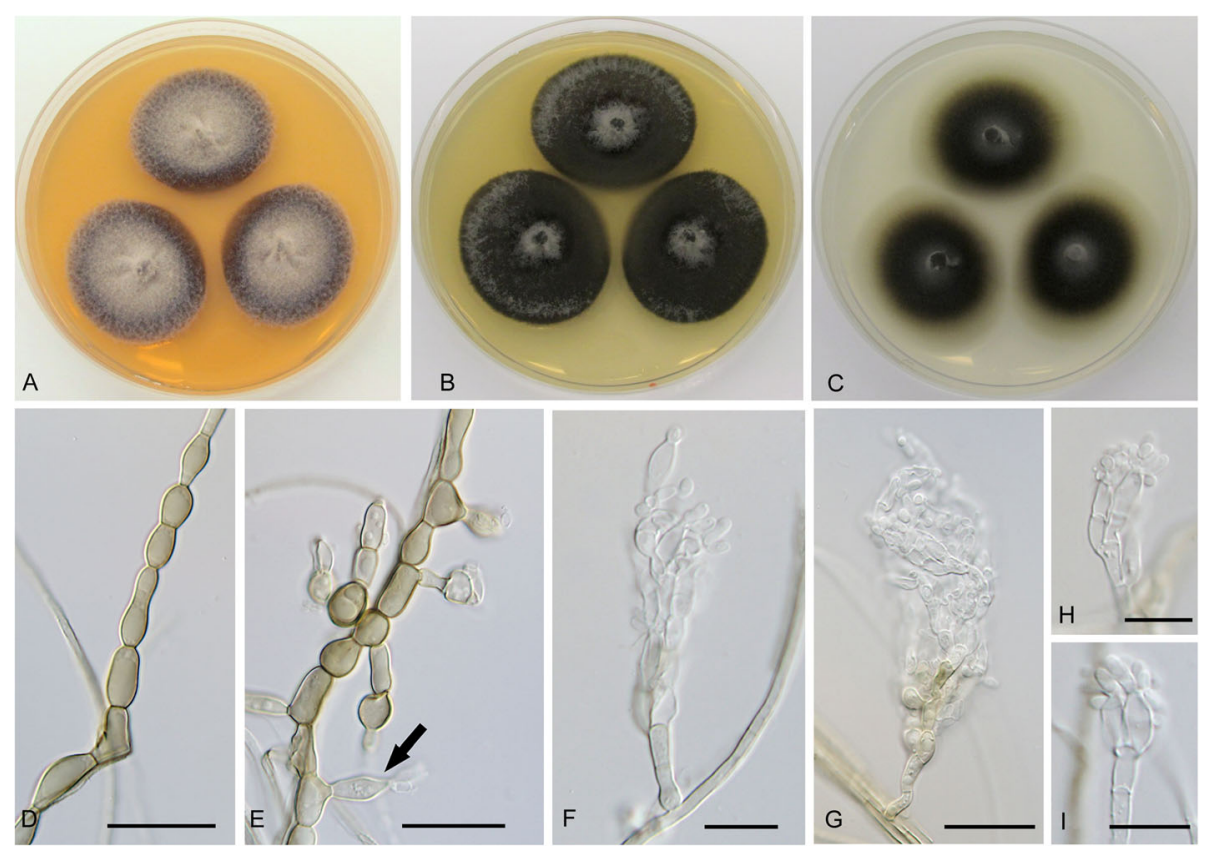

Fig. 12 Cadophora cf. orchidicola (isolate P1854). a - c, 15-day-old colonies on MEA, PDA, and CMA, respectively. d - e, inflated hyphal segments (arrow points to a conidiogenous cell). $\mathbf{f}-\mathbf{i}$, conidiophores with fascicles of conidiogenous cells. Bars: D - G = $20 \mu \mathrm{m}, \mathrm{H}-\mathrm{I}=10 \mu \mathrm{m}$

fascicles. Conidiogenous cells cylindrical, slightly conical or inflated in lower part, presumably enteroblastic, but without apparent collarette, 6-9.5(-12) $\mu \mathrm{m}$ long and $2.5-5 \mu \mathrm{m}$ wide. Conidia produced sympodially, ellipsoid, hyaline, with smooth wall, $3-5 \times 1.5-2.5 \mu \mathrm{m}$ wide, forming clusters of $2-4$ on the top of the conidiogenous cell, the mean conidium length/width ratio 1.9:1.

Specimens examined: Bulgaria, Begunovtsi, $42.70 \mathrm{~N}$ 022.83 E, $770 \mathrm{~m}$ a.s.l., endophytic in roots of $M$. erraticum, 14 May 2013, T. Ali \& S. Ploch [isol. K. Glynou] (FR 0255161; P1854 = CBS 146372 - cultures).

Note: The identification of this clade formed by 23 isolates sequenced in this study is only tentative due to ambiguous evidence from phenotypic and molecular data. A representative strain of this clade intensively sporulating in culture (P1854) was markedly similar to the description of $C$. orchidicola. This species originally described from roots of the orchid Calypso bulbosa was characterized as producing "slightly swollen conidiogenous cells" and "terminal conidia produced sympodially" without apparent scars (Currah et al. 1987). Our observations confirmed phialide-like conidiogenous cells, but without distinct collarette; i.e. we were not able to identify the exact mode of conidiogenesis. Also the dimensions of conidia were almost identical to those provided by Currah et al. (1987), i.e., 3-5(-7) × 1-3 $\mu \mathrm{m}$. However, reliable identification based on sequence data was not possible. Although the ex-type strain UAMH 5422 is available, C. orchidicola is not represented by any extype sequence in GenBank. Instead, the database contains more than 380 sequences of Cadophora/Leptodontidium orchidicola. BLAST searches performed with representative sequences from this clade indicated multiple matches with similarities of 94.5-99.5\%, indicating substantial inconsistency in species delimitation.

Cadophora variabilis Koukol \& Maciá-Vicente, sp. nov. - Figs. 1, 13.

MycoBank: MB 834829.

Etymology: After the production of conidia of variable shapes.

Diagnosis: Morphologically very similar to C. antarctica, but with ramoconidia up to $25.5 \mu \mathrm{m}$ and conidia up to $27 \mu \mathrm{m}$ long.

Type: Croatia: Jasenice, $44.24 \mathrm{~N}, 015.54 \mathrm{E}, 760 \mathrm{~m}$ a.s.l., endophytic in roots of $M$. perfoliatum, 4 May 2013, $T$. Ali [isol. K. Glynou] (FR 0255203 - holotype; P1176= CBS 146360 - ex-type cultures; GenBank accessions: ITS $=$ KT268493, LSU $=$ MK539845, tef1 $-\alpha=$ MK550890, $r p b 2=$ MN367276).

Description: In culture - Colonies on MEA reaching 9$10 \mathrm{~mm}$ diam after $10 \mathrm{~d}$, on PDA and CMA reaching 10$11 \mathrm{~mm}$ and $9-10 \mathrm{~mm}$ diam, respectively. Mycelium 


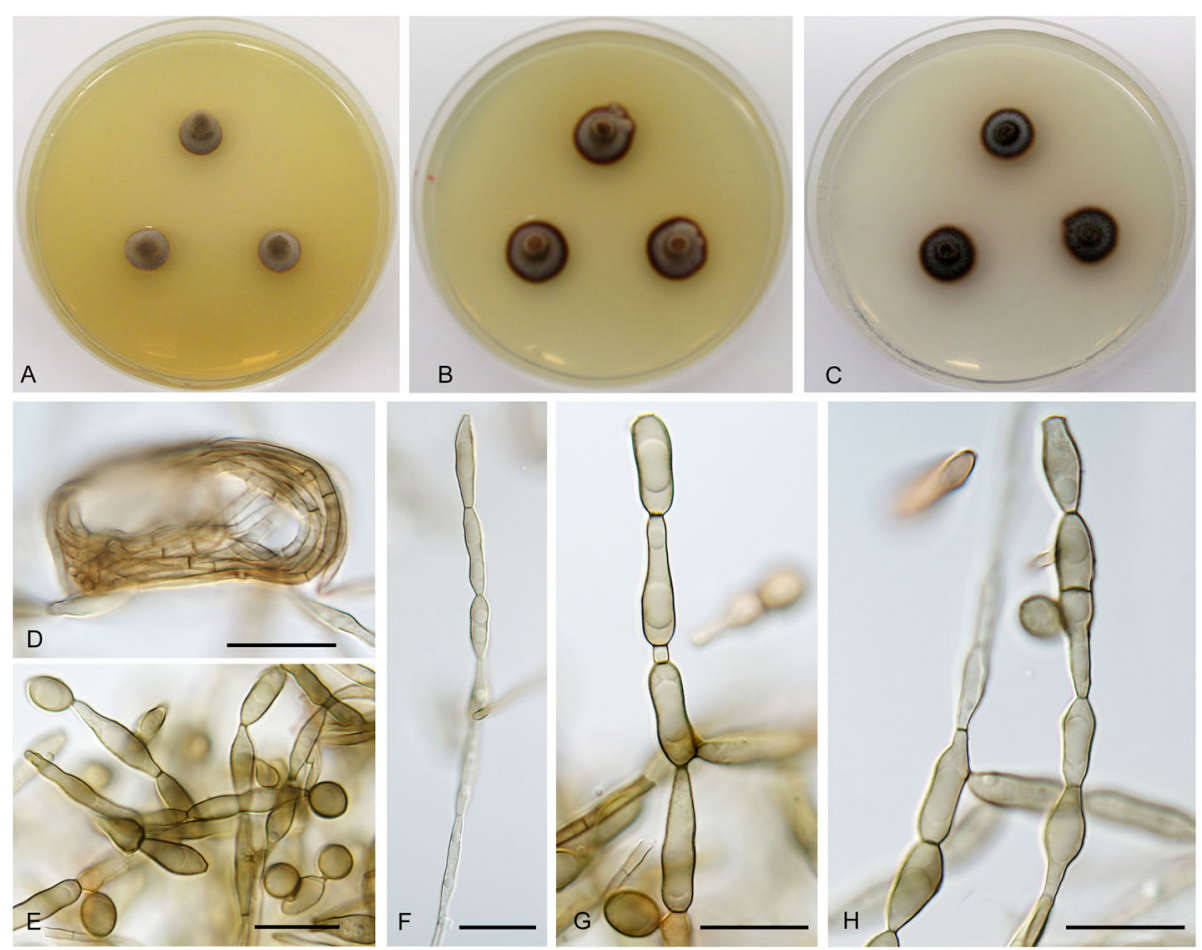

Fig. 13 Cadophora variabilis (CBS 146360 - ex-type culture). a - c, 15-day-old colonies on MEA, PDA, and CMA, respectively. d, hyphal coil. e - h, conidial chains. Bars: $20 \mu \mathrm{m}$

septate, hyphae hyaline to dark brown, rarely forming coils $55 \mu \mathrm{m}$ diam, hyphae $2-3 \mu \mathrm{m}$ wide. Conidiophores not developed. Conidiogenous cells integrated, terminal, holoblastic, monoblastic, smooth, cylindrical, 3-5 $\mu \mathrm{m}$ wide. Ramoconidia holoblastic non-septate, pale brown to hyaline, smooth, straight, fusiform, frequently constricted in the middle, (16-)18-23.5(-25.5) × 5-7.5 $\mu \mathrm{m}$. Conidia pale brown to hyaline, smooth, mostly nonseptate (rarely 1-septate), rather variable in shape, subglobose to pyriform, obovate or fusiform, with a distinct constriction in the middle when elongated, forming chains (rarely with cylindrical connective or aberrant conidium), (8.5-)11-21 $(-27) \times 5.5-9 \mu \mathrm{m}$, mean conidium length/width ratio $2.2: 1$.

Additional material examined. Spain: Puebla de Don Fadrique, $38.04 \mathrm{~N}, 002.48 \mathrm{~W}, 1630 \mathrm{~m}$ a.s.l., endophytic in roots of M. perfoliatum, 2 May 2013, J.G. Maciá-Vicente [isol. K. Glynou] (FR 0255145; P1331 = CBS 146364 - cultures).

Note: Cadophora variabilis is very similar to C. antarctica, which also produces chains of ramoconidia and conidia on holoblastic conidiogenous cells. However, the ramoconidia and conidia of $C$. variabilis are larger than those of $C$. antarctica. Interestingly, these two species are very distant in the phylogeny suggesting that this similar morphology has resulted from convergence.

\section{DISCUSSION}

The roots of non-mycorrhizal Brassicaceae harbour an unexpected diversity of helotialean endophytes, among other fungi from diverse lineages (Glynou et al. 2016, 2018a). Our screening of only a few plant species, mainly from the genus Microthlaspi, yielded seven Cadophora species new to science and six additional lineages that also are likely novel species, altogether representing more than a half of the species included so far in the genus. Our results clearly show that Cadophora as currently circumscribed is paraphyletic, sharing a common ancestor with species recognized in other genera, and evolved into diverse morphologies and ecological preferences while simultaneously converging in many of their phenotypic characters. Even though most of the species described herein appear to be somewhat rare, they often display relatively wide distribution ranges across the northern hemisphere, mainly in Europe. Some species, however, may be strictly endemic with a very limited distribution range.

\section{Taxonomy}

The paraphyly we found in the genus Cadophora had been previously reported by Day et al. (2012), who identified Cadophora species distributed across three different clades based on an ITS phylogeny: one containing Cadophora species together with species in Phialocephala and 
Mollisia (clade A in Day et al. 2012), another also containing species of Chloridium and Meliniomyces (clade B), and a third comprising most Cadophora species, including the type of $C$. fastigiata (clade C). With the combination of $C$. hiberna into Phialocephala by Day et al. (2012), and of $C$. finlandica into Hyaloscypha by Fehrer et al. (2019), the genus apparently became monophyletic. This was supported by recent studies (Linnakoski et al. 2018; Bien and Damm 2020) in which phylogenies were biased by excluding genera closely related to Cadophora. The final dataset assembled in our study not only expands several times the clade C of Day et al. (2012), but also provides a clear support for the paraphyly of Cadophora. Species of the genera Collembolispora, Helgardia, Mycochaetophora, Oculimacula, Rhexocercosporidium, and Rhynchosporium, are interspersed between the clades formed by Cadophora species (Fig. 1). The ITS sequences alone are not enough to differentiate species within this group, and even single coding-gene phylogenies may result in misleading information. Interestingly, the same gene may have different taxonomic value for different Cadophora clades. Whilst Walsh et al. (2018) found clear differentiation of C. interclivum and C. meredithiae based on the $\beta$-tubulin gene, Bien and Damm (2020) suspected the same gene of having paralogues in C. bubakii, C. obscura and C. viticola. The phylogenetic placement of Graphium rubrum, represented by a sequence from the ex-type strain CBS 210.34, next to C. malorum, is most probably misleading as that strain does not seem to truly originate from the type (Harrington et al. 2001; Harrington and McNew 2003).

Based on our multi-gene phylogeny, we consider that Cadophora s. str. forms a distinct basal clade comprising the type of the genus, C. fastigita (represented by strain CBS 307.48), together with C. melinii, C. margaritata, $C$. novi-eboraci, C. orientoamericana, C. viticola, C. ramosa, and C. ferruginea. Although another C. fastigiata strain, DAOM 225754, appears in a distant position with reference to Cadophora s. str., we consider that $C$. fastigiata strain CBS 307.48 better represents the type species. This strain was isolated from blue-stained wood in Sweden, just like the species type (Lagerberg et al. 1927), and Schol-Schwartz (1970) noted no morphological differences between both cultures. Later studies also used this strain as a representative of C. fastigiata (Bien and Damm 2020) or even have considered it, erroneously, derived from the type (Travadon et al. 2015; Crous et al. 2017; Linnakoski et al. 2018).

The paraphyly in the genus Cadophora, evidenced here, deserves addressing, possibly most efficiently by splitting the genus into a minimum of three genera to accommodate the clades containing: (1) Cadophora s. str.; (2) C. orchidicola and C. echinata (while simultaneously combining and taking the generic name from Collembolispora); and (3) C. interclivum, C. meredithiae,
C. luteo-olivacea, C. malorum, and C. helianthi. In this context, C. antarctica, C. lacrimiformis and C. fascicularis should be combined into Mycochaetophora. Nevertheless, we refrain from undertaking this drastic restructuring here, owing to the lack of established, morphological generic concepts, i.e., concerning the corresponding type species that belong to the Cadophora $s$. lat. relationship-as well as of sequence data of further sexual species that would need to be considered for such a task. The creation of new generic concepts without a more comprehensive sampling of species could well lead to their future recognition as synonyms, a situation we want to avoid. Further efforts aimed at the discovery of additional new species, above all with sexual morphs, the sequencing of molecular markers from type specimens, and the epitypification, will be necessary for a robust establishment of the phylogenetic relationships among the lineages thus far constituting Cadophora and allied genera.

\section{Relationships between morphological and phylogenetical data}

Day et al. (2012) hypothesized that conidiogenesis in Cadophora evolved via simplification of more complex ancestral phialide arrangements, including sclerotiumlike structures formed by compact multiple phialides that could serve as diaspores produced on wood surfaces. This evolution could have depended on a higher reliance on conidia for dispersal, and led to the near absence of conidia in C. orchidicola (Day et al. 2012), which we corroborated for a large number of isolates related to this species. Our data show a distinct evolution of conidiogenesis across clades, with Cadophora s. str. species having evolved from an ancestor with phialidic ontogeny of conidia. Cadophora s. str. species thereby differ from the ancestor of other Cadophora species, whose conidia were produced holoblastically. Phialidic conidiogenesis appears to have evolved multiple times, giving rise to species in the C. gregata, C. luteo-olivacea, and C. malorum clade (node 6 in Fig. 2). A notable case within this clade is C. obovata, which seems to have lost the ability to form further conidia enteroblastically after the first holoblastic conidium is formed. On the contrary, C. orchidicola, placed among species producing only holoblastic conidia, has conidiogenesis of an unclear type (Harrington and McNew 2003) showing some similarity to phialidic conidiogenesis. Conidiogenesis thus appears as an important character not only for the identification of Cadophora species based on morphology, but also for the evolution of the phenotype. However, in our study many isolates failed to sporulate even after incubation for more than one year at $4{ }^{\circ} \mathrm{C}$, which appears to be required for conidiophore formation in some Cadophora and related species (Addy et al. 2005). 
Other microscopical characters such as the characteristics of conidia are rather variable across the Cadophora groups, thus offering limited diagnostic potential, as is the case with macroscopical culture traits such as the shape or texture of the mycelium that are strongly pleomorphic in this group. Overall, morphological characteristics are a poor proxy for phylogenetic affinities in Cadophora. The limited availability of diagnostic morphological traits in this and other DSE groups has been responsible for the long-standing difficulty in establishing their systematic positions, that varied across distant taxonomic categories (Gams 2000; Jumpponen 2001; Harrington and McNew 2003; Addy et al. 2005).

\section{Ecological preferences}

The variability in morphological traits makes it difficult to infer the ecological reasons behind the evolution of particular characters, with the only exception of the aquatic genus $\mathrm{Col}$ lembollispora, with branched conidia typical of ingoldian fungi (Dix and Webster 1995). However, the phialidic synanamorph of Collembolispora barbata provides a morphological connection to members of Cadophora. The first Cadophora species was described as a wood-colonizing fungus, and today species in the genus are still frequently isolated from this substrate (Lagerberg et al. 1927; Bills 2004; Gramaje et al. 2011, 2015; Bien and Damm 2020). Our results support such a substrate preference for species of Cadophora s. str., but show a widespread ability to colonize living plant hosts in the remaining clades - particularly roots as a result of the focus of this study. This ability has been confirmed experimentally for several isolates included in our study, representing C. cf. interclivum (P1686, P1866), C. cf. orchidicola (P1940), and C. variabilis (P1176, P1331), all of which have been shown able to colonize roots from Brassicaceae and Poaceae hosts without eliciting disease symptoms (Kia et al. 2017, 2018, 2019). Such a root-colonizing habit could indicate that the ancestor of Cadophora s. lat. evolved towards a symbiotic lifestyle when split from the ancestor of Cadophora s. str. However, reaching definite conclusions about the ecological preferences of the different species is not possible based only on a few representative isolates of many species. In addition, the ability to colonize multiple niches is a common phenomenon in fungi (Selosse et al. 2018), so that the diversity of substrates of Cadophora species may just reflect ecological plasticity.

\section{Distribution patterns}

Comparisons of ITS sequences with data available in public databases showed that many of the focal species in this study are related to fungi widely distributed across the Northern Hemisphere, although in most cases nearly identical sequences were exclusively found within Europe. This is in line with previous observations on the distribution of other helotialean DSE in the
Phialocephala fortinii and Acephala applanata species complexes (Grünig et al. 2001; Piercey et al. 2004; Queloz et al. 2011), which show a widespread distribution in northern temperate zones. The absence of similar sequences in the Southern Hemisphere could be caused by biases in the geographic representation of fungal ITS sequence data in GenBank, with several Southern Hemisphere countries being underrepresented as compared to countries in the Northern one. This possibility is, indeed, suggested by a higher representation in the Southern Hemisphere of UNITE SH assigned to Cadophora sp. 1, Cadophora sp. 4, Cadophora sp. 5, C. echinata, C. cf. interclivum, C. ferruginea, C. luteo-olivacea, $C$. cf. meredithiae, and $C$. variabilis (Table S1). However, northern countries such as the USA and China, amongst the most represented by fungal ITS sequences in GenBank, did not yield as many BLAST matches as European countries did, thus adding support to the distribution patterns we observed.

Just considering our isolates, all species with more than two isolates had representatives at separate locations over Europe, while often co-existing in the same site with isolates of other Cadophora species. This reproduces the observations of Grünig et al. (2002) and Piercey et al. (2004) on $P$. fortinii, who found individuals from separate locations were more closely related to one another than were individuals collected a few metres apart, and also agrees with similar results with several lineages of dominant root endophytes (Glynou et al. 2017). The mechanisms for such long distance dispersal are unknown, for example whether this is achieved via conidial dispersion or via transportation within plant material as microsclerotia or other diaspores (Currah et al. 1993). We did not observe a correlation between the production of conidia in culture and the breadth of the species distribution ranges, e.g. $C$. orchidicola barely produces conidia but has widespread occurrences, and $C$. fascicularis which produces numerous conidia but has a restricted distribution. In contrast to the wide distribution of most species, $C$. fascicularis, $C$. ferruginea, Cadophora sp. 3, Cadophora sp. 5, and Mycochaetophora sp. show very restricted distribution ranges both worldwide and across Europe, with occurrences in only one or a few close locations. Given the scarcity of isolates and identical sequences available for these species, it is difficult to conclude that the restricted distributions of these species are due to true endemism, with species adapted only to very local conditions, or due to rarity that precludes their capture in ecological studies by current sampling methods. This question will only be settled upon by further sampling activities and descriptions of fungal diversity worldwide.

\section{CONCLUSIONS}

Our dataset is probably the most comprehensive considering currently recognized Cadophora species. It shows 
an unexpectedly high diversity of species in roots of Microthlaspi spp., which led to the description of seven species new to science in this study and enabled a new and a more complete view of the life history of members of this helotialean genus to be provided. Our results also show current knowledge gaps in the phylogenetic relationships within Cadophora and with other, related lineages. Further sampling efforts to discover new species, as well as further descriptions and sequence data from species types, are necessary to resolve the systematics of this fascinating group of fungi.

\section{Supplementary information}

Supplementary information accompanies this paper at https://doi.org/10. 1186/s43008-020-00036-w

Additional file 1: Fig. S1. Clustering of the focal isolates of this study into operational taxonomic units (OTUs) based on pairwise ITS rDNA sequence similarity at 97, 98 and 99\%. The trees represent a subset of the phylogeny shown in Fig. 1, including only the isolates used in this study, with tip labels and colors representing the OTU grouping of isolates at each similarity threshold. The OTU clustering was performed as described in Glynou et al. (2016), representing a standard procedure in fungal community ecology studies. Fig. S2. Ancestral character state reconstruction in Cadophora and allied species for all morphological and ecological characteristics considered in this study. Fig. S3. Principal component analysis (PCA) ordination of isolates according to their quantitative morphological characters, including those removed from Fig. 3. Fig. S4. Distribution of all quantitative characters considered in this study across the isolates' phylogeny. Colors next to tree tips indicate the isolates' species (see color key). Fig. S5. Potential worldwide distribution of all the Cadophora species target of this study as inferred by BLAST comparisons against the NCBI GenBank's nucleotide database (nt), and selected high-throughput ITS amplicon sequencing datasets available at the sequence reads archive (SRA; Table S3). Each map shows the BLAST search results for all the isolates within each species. Points represent the geographic locations of BLAST matches, either from the nt or the SRA database (indicated by point shape). Only BLAST matches with percent identity above $97 \%$ are shown, with points color indicating percent identity value.

Additional file 2: Table S1. Details of the fungal isolates used in this study, including accession numbers, morphological characters, and data on the geographical location, climate, and soil conditions at the site of origin. Table S2. Details of the reference strain sequences included in the phylogenetic analysis. Table S3. NCBI data sets of reference ITS sequences used for BLAST comparisons. Table S4. Phylogenetic conservation among quantitative traits based on Blomberg's $K$.

\section{Acknowledgments}

We thank Claudia Nesselhauf for her assistance in the curation of the isolates' dry cultures. This study was supported by LOEWE (Landes-Offensive zur Entwicklung Wissenschaftlich-ökonomischer Exzellenz) of the state of Hesse and was conducted within the framework of the Cluster for Integrative Fungal Research (IPF). J.G.M.-V. acknowledges support from the German Research Foundation under grant MA7171/1-1. O.K. thanks to institutional support for science and research provided by the Ministry of Education, Youth and Sports of the Czech Republic.

\section{Adherence to national and international regulations}

All the isolates were obtained following the regulations of the Convention on Biological Diversity.

\section{Authors' contributions}

JGM-V conceived the study, obtained the DNA sequences, and performed the data analyses. JGM-V and OK performed the morphological characterization of the isolates and the phylogenetic analyses. OK described the new species. JGM-V and MP contributed materials and reagents. All authors interpreted the results. JGM-V and OK wrote the first draft of the manuscript, and all authors contributed to the final version. The authors read and approved the final manuscript.

\section{Funding}

This study was supported by LOEWE (Landes-Offensive zur Entwicklung Wissenschaftlich-ökonomischer Exzellenz) of the state of Hesse and was conducted within the framework of the Cluster for Integrative Fungal Research (IPF). J.G.M.-V. acknowledges support from the German Research Foundation under grant MA7171/1-1. O.K. thanks to institutional support for science and research provided by the Ministry of Education, Youth and Sports of the Czech Republic.

\section{Availability of data and materials}

Living cultures of all isolates have been deposited in the IPF fungal culture collection hosted at Goethe University Frankfurt, and a representative subset of the isolates were also deposited in the CBS culture collection (Westerdijk Fungal Biodiversity Institute, Utrecht, The Netherlands). Dried cultures were deposited in the Herbarium Senckenbergianum Frankfurt am Main (FR). The DNA alignments and the phylogenetic trees are available at TreeBASE under accession S25942. All data and code used in this study is available online at https://doi.org/10.6084/m9.figshare.12287816.

Ethics approval and consent to participate

Not applicable.

\section{Consent for publication}

Not applicable.

\section{Competing interests}

The authors declare no competing interests.

\section{Author details}

${ }^{1}$ Institute of Ecology, Evolution and Diversity, Goethe University Frankfurt, Max-von-Laue-Str. 13, 60438 Frankfurt am Main, Germany. ${ }^{2}$ Integrative Fungal Research Cluster (IPF), Frankfurt am Main, Germany. ${ }^{3}$ Department of Botany, Faculty of Science, Charles University, Benátská 2, 12801 Praha 2, Czech Republic.

Received: 16 March 2020 Accepted: 11 June 2020

Published online: 11 August 2020

\section{References}

Addy HD, Piercey MM, Currah RS (2005) Microfungal endophytes in roots. Canadian Journal of Botany 83:1-13. https://doi.org/10.1139/b04-171

Almario J, Jeena G, Wunder J, Langen G, Zuccaro A et al (2017) Root-associated fungal microbiota of nonmycorrhizal Arabis alpina and its contribution to plant phosphorus nutrition. In: Proceedings of the National Academy of Sciences, p 201710455. https://doi.org/10.1073/pnas.1710455114

Ashrafi S, Knapp DG, Blaudez D, Chalot M, Maciá-Vicente JG et al (2018) Inhabiting plant roots, nematodes and truffles - Polyphilus, a new helotialean genus with two globally distributed species. Mycologia 110:286299. https://doi.org/10.1080/00275514.2018.1448167

Becker OS, code by RA, Minka ARWR version by RBE by TP, Deckmyn A (2018) Maps: draw geographical maps

Berthelot C, Leyval C, Foulon J, Chalot M, Blaudez D (2016) Plant growth promotion, metabolite production and metal tolerance of dark septate endophytes isolated from metal-polluted poplar phytomanagement sites. FEMS Microbiology Ecology 92:fiw144. https://doi.org/10.1093/femsec/fiw144

Bien S, Damm U (2020) Arboricolonus simplex gen. et sp. nov. and novelties in Cadophora, Minutiella and Proliferodiscus from Prunus wood in Germany. MycoKeys 63:119-161. https://doi.org/10.3897/mycokeys.63.46836

Bills GF (2004) Cadophora hiberna sp. nov., a winter-fruiting helotialean anamorph from wood of Robinia pseudoacacia and forest soil. Memoirs of the New York Botanical Garden 89:113-124

Bizabani C, Dames J (2015) Effects of inoculating Lachnum and Cadophora isolates on the growth of Vaccinium corymbosum. Microbiological Research 181:68-74. https://doi.org/10.1016/j.micres.2015.08.005 
Blomberg SP, Garland T, Ives AR (2003) Testing for phylogenetic signal in comparative data: behavioral traits are more labile. Evolution 57:717-745. https://doi.org/10.1111/j.0014-3820.2003.tb00285.x

Carbone I, Kohn LM (1999) A method for designing primer sets for speciation studies in filamentous ascomycetes. Mycologia 91:553-556. https://doi.org/ $10.2307 / 3761358$

Chavent M, Kuentz-Simonet V, Labenne A, Saracco J (2014) Multivariate analysis of mixed data: the PCAmixdata R package. arXiv 132:1411.4911

Conant NF (1937) The occurrence of a human pathogenic fungus as a saprophyte in nature. Mycologia 29:597-598

Crous PW, Carnegie AJ, Wingfield MJ, Sharma R, Mughini G et al (2019) Fungal planet description sheets: 868-950. Persoonia : Molecular Phylogeny and Evolution of Fungi 42:291-473. https://doi.org/10.3767/persoonia.2019.42.11

Crous PW, Wingfield MJ, Burgess TI, Carnegie AJ, Hardy GESJ et al (2017) Fungal planet description sheets: 625-715. Persoonia : Molecular Phylogeny and Evolution of Fungi 39:270-467. https://doi.org/10.3767/persoonia.2017.39.11

Currah RS, Sigler L, Hambleton S (1987) New records and new taxa of fungi from the mycorrhizae of terrestrial orchids of Alberta. Canadian Journal of Botany 65:2473-2482. https://doi.org/10.1139/b87-336

Currah RS, Tsuneda A, Murakami S (1993) Morphology and ecology of Phialocephala fortinii in roots of Rhododendron brachycarpum. Canadian Journal of Botany 71:1639-1644. https://doi.org/10.1139/b93-199

Darriba D, Taboada GL, Doallo R, Posada D (2012) jModelTest 2: more models, new heuristics and parallel computing. Nature Methods 9:772-772. https://doi.org/10.1038/nmeth.2109

Day MJ, Hall JC, Currah RS (2012) Phialide arrangement and character evolution in the helotialean anamorph genera Cadophora and Phialocephala. Mycologia 104:371-381. https://doi.org/10.3852/11-059

Dix NJ, Webster J (1995) Fungal ecology. Chapman \& Hall, London

Durán P, Jorquera M, Viscardi S, Carrión VJ, Mora ML et al (2017) Screening and characterization of potentially suppressive soils against Gaeumannomyces graminis under extensive wheat cropping by Chilean indigenous communities. Frontiers in Microbiology 8. https:/doi.org/10.3389/fmicb.2017.01552

Ekanayaka AH, Hyde KD, Gentekaki E, McKenzie EHC, Zhao Q et al (2019) Preliminary classification of Leotiomycetes. Mycosphere 10:310-489

Fehrer J, Réblová M, Bambasová V, Vohník M (2019) The root-symbiotic Rhizoscyphus ericae aggregate and Hyaloscypha (Leotiomycetes) are congeneric: phylogenetic and experimental evidence. Studies in Mycology 92:195-225. https://doi.org/10.1016/j.simyco.2018.10.004

Fitter AH (2005) Darkness visible: reflections on underground ecology. Journal of Ecology 93:231-243. https://doi.org/10.1111/j.0022-0477.2005.00990.x

Gams W (2000) Phialophora and some similar morphologically little-differentiated anamorphs of divergent ascomycetes. In: Studies in mycology, pp 187-200

Glynou K, Ali T, Buch A-K, Kia SH, Ploch S et al (2016) The local environment determines the assembly of root endophytic fungi at a continental scale. Environmental Microbiology 18:2418-2434. https://doi.org/10.1111/14622920.13112

Glynou K, Ali T, Kia SH, Thines M, Maciá-Vicente JG (2017) Genotypic diversity in root-endophytic fungi reflects efficient dispersal and environmental adaptation. Molecular Ecology 26:4618-4630. https://doi.org/10.1111/mec.14231

Glynou K, Nam B, Thines M, Maciá-Vicente JG (2018a) Facultative root-colonizing fungi dominate endophytic assemblages in roots of nonmycorrhizal Microthlaspi species. New Phytologist 217:1190-1202. https:/doi.org/10.1111/nph.14873

Glynou K, Thines M, Maciá-Vicente JG (2018b) Host species identity in annual Brassicaceae has a limited effect on the assembly of root-endophytic fungal communities. Plant Ecology and Diversity 11:569-580. https://doi.org/10. 1080/17550874.2018.1504332

Gramaje D, Mostert L, Armengol J (2011) Characterization of Cadophora luteo-olivacea and C. melinii isolates obtained from grapevines and environmental samples from grapevine nurseries in Spain. Phytopathologia Mediterranea 50:112-126

Gramaje D, Mostert L, Armengol J (2015) Cadophora viticola D. Gramaje, L. Mostert \& Armengol, sp. nov. Persoonia 34:243

Greenleaf MA, Korf RP (1980) Mollisia in Macaronesia: an exercise in frustration. Mycotaxon 10:459-472

Grünig CR, Sieber TN, Holdenrieder O (2001) Characterisation of dark septate endophytic fungi (DSE) using inter-simple-sequence-repeat-anchored polymerase chain reaction (ISSR-PCR) amplification. Mycological Research 105:24-32. https://doi.org/10.1017/S0953756200003658

Grünig CR, Sieber TN, Rogers SO, Holdenrieder O (2002) Spatial distribution of dark septate endophytes in a confined forest plot. Mycological Research 106: 832-840. https://doi.org/10.1017/S0953756202005968
Hacquard S, Kracher B, Hiruma K, Münch PC, Garrido-Oter R et al (2016) Survival trade-offs in plant roots during colonization by closely related beneficial and pathogenic fungi. Nature Communications 7:11362. https://doi.org/10.1038/ ncomms 11362

Harrington TC, McNew D, Steimel J, Hofstra D, Farrell R (2001) Phylogeny and taxonomy of the Ophiostoma piceae complex and the Dutch elm disease fungi. Mycologia 93:111-136. https://doi.org/10.1080/00275514.2001. 12061284

Harrington TC, McNew DL (2003) Phylogenetc analysis places the Phialophora-like anamorph genus Cadophora in the Helotiales. Mycotaxon 87:e151

Hopple JS Jr, Vilgalys R (1994) Phylogenetic relationships among coprinoid taxa and allies based on data from restriction site mapping of nuclear rDNA. Mycologia 86:96-107. https://doi.org/10.2307/3760723

Jaklitsch W, Baral HO, Lücking R, Lumbsch HT, Frey W (2016) Syllabus of plant families-a. Engler's syllabus der Pflanzenfamilien part 1/2, 13th edn $322 \mathrm{pp}$

Johnston PR, Quijada L, Smith CA, Baral HO, Hosoya T et al (2019) A multigene phylogeny toward a new phylogenetic classification of Leotiomycetes. IMA Fungus 10:1. https://doi.org/10.1186/s43008-019-0002-x

Jumpponen A (2001) Dark septate endophytes - are they mycorrhizal? Mycorrhiza 11:207-211. https://doi.org/10.1007/s005720100112

Katoh K, Standley DM (2013) MAFFT multiple sequence alignment software version 7: improvements in performance and usability. Molecular Biology and Evolution 30:772-780. https://doi.org/10.1093/molbev/mst010

Kearse M, Moir R, Wilson A, Stones-Havas S, Cheung M et al (2012) Geneious basic: an integrated and extendable desktop software platform for the organization and analysis of sequence data. Bioinformatics 28:1647-1649. https://doi.org/10.1093/bioinformatics/bts199

Kia SH, Glynou K, Nau T, Thines M, Piepenbring M et al (2017) Influence of phylogenetic conservatism and trait convergence on the interactions between fungal root endophytes and plants. ISME Journal 11:777-790. https://doi.org/10.1038/ismej.2016.140

Kia SH, Jurkechova M, Glynou K, Piepenbring M, Maciá-Vicente JG (2018) The effects of fungal root endophytes on plant growth are stable along gradients of abiotic habitat conditions. FEMS Microbiology Ecology 94:fix162. https:// doi.org/10.1093/femsec/fix162

Kia SH, Pallesch S, Piepenbring M, Maciá-Vicente JG (2019) Root endophytic fungi show low levels of interspecific competition in planta. Fungal Ecology 39: 184-191. https://doi.org/10.1016/j.funeco.2019.02.009

Knapp DG, Németh JB, Barry K, Hainaut M, Henrissat B et al (2018) Comparative genomics provides insights into the lifestyle and reveals functional heterogeneity of dark septate endophytic fungi. Scientific Reports 8:6321. https://doi.org/10.1038/s41598-018-24686-4

Kõljalg U, Larsson K-H, Abarenkov K et al (2005) UNITE: a database providing web-based methods for the molecular identification of ectomycorrhizal fungi. New Phytologist 166:1063-1068. https://doi.org/10.1111/j.1469-8137. 2005.01376.x

Kõljalg U, Nilsson RH, Abarenkov K, Tedersoo L, Taylor AFS et al (2013) Towards a unified paradigm for sequence-based identification of fungi. Molecular Ecology 22:5271-5277. https://doi.org/10.1111/mec.12481

Lagerberg T, Lundberg G, Melin E (1927) Biological and practical researches into blueing in pine and spruce (gen. Cadophora). Sven Skvgsr Tidskr 25:145172

Legendre P, Legendre LFJ (2012) Numerical ecology, volume 24, third edition, 3rd edn. Elsevier, Amsterdam

Linnakoski R, Kasanen R, Lasarov I, Marttinen T, Oghenekaro AO et al (2018) Cadophora margaritata sp. nov. and other fungi associated with the longhorn beetles Anoplophora glabripennis and Saperda carcharias in Finland. Antonie Van Leeuwenhoek 111:2195-2211. https://doi.org/10.1007/s10482-018-1112-y

Maciá-Vicente JG, Glynou K, Piepenbring M (2016) A new species of Exophiala associated with roots. Mycological Progress 15:1-12. https://doi.org/10.1007/ s11557-016-1161-4

Maciá-Vicente JG, Nam B, Thines M (2020) Root filtering, rather than host identity or age, determines the composition of root-associated fungi and oomycetes in three naturally co-occurring Brassicaceae. Soil Biology and Biochemistry 146:107806. https://doi.org/10.1016/j.soilbio.2020.107806

Maciá-Vicente JG, Shi Y-N, Cheikh-Ali Z, Grün P, Glynou K et al (2018) Metabolomics-based chemotaxonomy of root endophytic fungi for natural products discovery. Environmental Microbiology 20:1253-1270. https://doi. org/10.1111/1462-2920.14072

Miller MA, Pfeiffer W, Schwartz T (2010) Creating the CIPRES science gateway for inference of large phylogenetic trees. In: 2010 gateway computing environments workshop (GCE). IEEE Computer Society, New Orleans, pp 1-8 
Oksanen J, Blanchet FG, Kindt R, Legendre P, Minhin PR et al (2015) Vegan: community ecology package

Paradis E, Claude J, Strimmer K (2004) APE: analyses of phylogenetics and evolution in R language. Bioinformatics 20:289-290. https://doi.org/10.1093/ bioinformatics/btg412

Pärtel K, Baral H-O, Tamm H, Pöldmaa K (2017) Evidence for the polyphyly of Encoelia and Encoelioideae with reconsideration of respective families in Leotiomycetes. Fungal Diversity 82:183-219. https://doi.org/10.1007/s13225016-0370-0

Piercey MM, Graham SW, Currah RS (2004) Patterns of genetic variation in Phialocephala fortinii across a broad latitudinal transect in Canada. Mycological Research 108:955-964. https://doi.org/10.1017/ S0953756204000528

Queloz V, Sieber TN, Holdenrieder O, McDonald BA, Grünig CR (2011) No biogeographical pattern for a root-associated fungal species complex. Global Ecology and Biogeography 20:160-169. https://doi.org/10.1111/j.1466-8238. 2010.00589.x

R Core Team (2019) R: a language and environment for statistical computing. $R$ Foundation for Statistical Computing, Vienna

Revell $L J$ (2012) Phytools: an R package for phylogenetic comparative biology (and other things). Methods in Ecology and Evolution 3:217-223. https://doi. org/10.1111/j.2041-210X.2011.00169.X

Ronquist F, Teslenko M, van der Mark P, Ayres DL, Darling A et al (2012) MrBayes 3.2: efficient bayesian phylogenetic inference and model choice across a large model space. Systematic Biology 61:539-542. https://doi.org/10.1093/ sysbio/sys029

Schindelin J, Arganda-Carreras I, Frise E, Kaynig V, Longair M et al (2012) Fij: an open-source platform for biological-image analysis. Nature Methods 9:676682. https://doi.org/10.1038/nmeth.2019

Schol-Schwartz M (1970) Revision of the genus Phialophora (Moniliades). Persoonia 6:59-94

Selosse M-A, Schneider-Maunoury L, Martos F (2018) Time to re-think fungal ecology? Fungal ecological niches are often prejudged. New Phytologist 217: 968-972. https://doi.org/10.1111/nph.14983

Sieber TN (2002) Fungal root endophytes. In: plant roots: the hidden half. CRC Press, Boca Raton, pp 887-917

South A (2017) Rnaturalearth: world map data from natural earth

Stamatakis A (2014) RAxML version 8: a tool for phylogenetic analysis and postanalysis of large phylogenies. Bioinformatics 9:1312-1313. https://doi.org/10 1093/bioinformatics/btu033

Tanney JB, Douglas B, Seifert KA (2016) Sexual and asexual states of some endophytic Phialocephala species of Picea. Mycologia 108:255-280. https:// doi.org/10.3852/15-136

Thiergart T, Duran P, Ellis T, Vannier N, Garrido-Oter R et al (2019) Root microbiota assembly and adaptive differentiation among European Arabidopsis populations. Nature Ecology \& Evolution 4:122-131. https://doi. org/10.1038/s41559-019-1063-3

Travadon R, Lawrence DP, Rooney-Latham S, Gubler WD, Wilcox WF et al (2015) Cadophora species associated with wood-decay of grapevine in North America. Fungal Biology 119:53-66. https://doi.org/10.1016/f.funbio.2014.11.002

Tsuneda A, Chen MH, Currah RS (2001) Conidiomatal morphogenesis and pleomorphic conidiogenesis in Scleroconidioma sphagnicola. Mycologia 93: 1164-1173. https://doi.org/10.1080/00275514.2001.12063249

Walsh E, Duan W, Mehdi M, Naphri K, Khiste S et al (2018) Cadophora meredithiae and C. interclivum, new species from roots of sedge and spruce in a western Canada subalpine forest. Mycologia 110:201-214. https://doi.org/10.1080/ 00275514.2017 .1406748

Wickham H, Chang W, Henry L, Pedersen TL et al (2019) ggplot2: create elegant data Visualisations using the grammar of graphics

\section{Publisher's Note}

Springer Nature remains neutral with regard to jurisdictional claims in published maps and institutional affiliations.

Ready to submit your research? Choose BMC and benefit from:

- fast, convenient online submission

- thorough peer review by experienced researchers in your field

- rapid publication on acceptance

- support for research data, including large and complex data types

- gold Open Access which fosters wider collaboration and increased citations

- maximum visibility for your research: over $100 \mathrm{M}$ website views per year

At $\mathrm{BMC}$, research is always in progress.

Learn more biomedcentral.com/submissions 